\title{
Particle Size Measurements from the first Fundamentals of Ice Crystal Icing Physics Test in the NASA Propulsion Systems Laboratory
}

\author{
Michael C. King ${ }^{1}$ \\ NASA Glenn Research Center, Cleveland, Ohio, 44135 \\ and \\ William Bachalo ${ }^{2}$ and Andrzej Kurek ${ }^{3}$ \\ Artium Technologies, Inc., Sunnyvale, CA, 94085
}

This paper presents particle measurements by the Artium Technologies, Inc. Phase Doppler Interferometer and High Speed Imaging instruments from the first Fundamental Ice Crystal Icing Physics test conducted in the NASA Propulsion Systems Laboratory. The work focuses on humidity sweeps at a larger and a smaller median volumetric diameter. The particle size distribution, number density, and water content measured by the Phase Doppler Interferometer and High Speed Imaging instruments from the sweeps are presented and compared. The current capability for these two instruments to measure and discriminate ICI conditions is examined.

\section{Nomenclature}

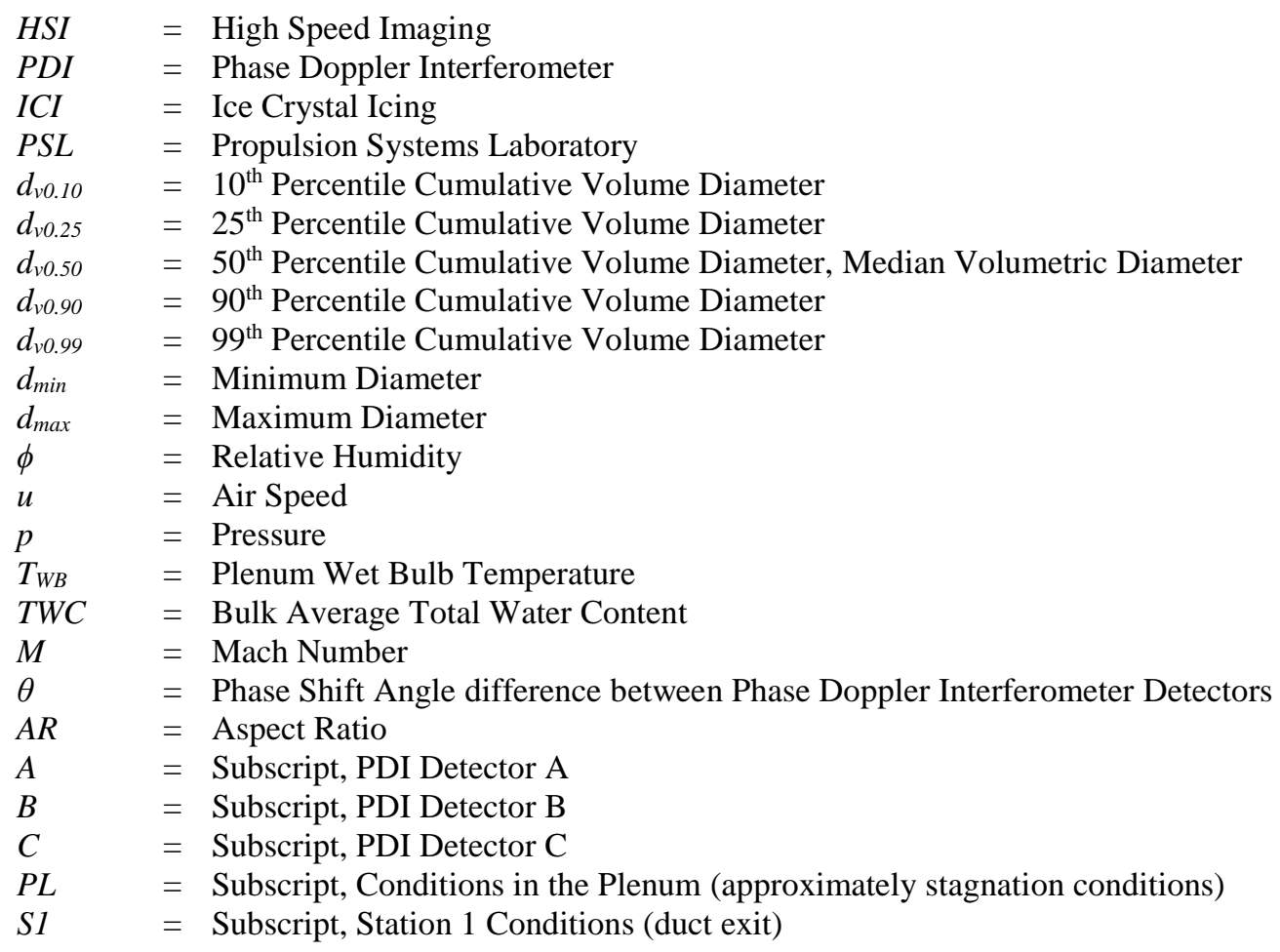

\footnotetext{
${ }^{1}$ Aerospace Engineer, Icing Branch, 21000 Brookpark Road, MS 11-2, Non-Member

${ }^{2}$ President, 470 Lakeside Drive, Unit C, Non-Member

${ }^{3}$ Research Engineer, 470 Lakeside Drive, Unit C, Non-Member
} 


\section{Introduction}

$\mathrm{T}$ HE ingestion of ice crystals into jet engines has been attributed to uncommanded jet engine power loss events during flight ${ }^{1-5}$, and has become a significant focus for research in the atmospheric icing community. As a result, NASA has been conducting ice crystal icing (ICI) studies in the the NASA Propulsion Systems Laboratory (PSL) to advance the community's understanding of the fundamental physics behind this aviation safety hazard ${ }^{6,7}$. The unique nature of ICI conditions has slowed cloud characterization in $\mathrm{PSL}^{8}$, but it has also stimulated the development of newer instrumentation to measure such conditions. The Artium Technologies, Inc. Phase Doppler Interferometer (PDI) and High Speed Imaging (HSI) instruments are two such examples that have been further developed as a result of the community's interest in ICI and funding support through the Small Business Innovative Research (SBIR) program.

This paper presents select cases of particle measurements acquired using the PDI and HSI instruments in PSL during the first Fundamentals of Ice Crystal Icing Physics Test conducted in March of 2016. The results presented herein examine the data acquired from the PDI and HSI during the test, comparing key parameters and evaluating the current capabilities of both instruments. The objective of this work is to increase understanding of the capabilities of these two instruments thereby allowing for improved characterization of mixed phase and ice crystal icing conditions.

\section{Experimental Description}

The first Fundamentals test generated mixed-phase ice accretions on a test article, a NACA 0012 airfoil wing section with a $267 \mathrm{~mm}$ chord, in a characterized cloud. The goal of the effort was to evaluate if PSL could be used for further Fundamentals tests by simulating conditions considered similar to those expected within a jet engine core during ICI conditions. Several instruments were used to characterize the mixed-phase cloud including systems from Droplet Measurement Technologies (DMT), Science Engineering Associates (SEA), the National Research Council of Canada (NRC), and NASA in addition to those from Artium Technologies, Inc. Besides the PDI and HSI from Artium Technologies, Inc., the DMT Cloud Droplet Probe (CDP) and Cloud Imaging Probe - Grey Scale (CIP-GS) were the only other particle sizing instruments used during the test. The results from the DMT instruments will be covered in a separate conference paper, Ref. 7.

\section{A. Phase Doppler Interferometer and High Speed Imaging Instruments}

The Artium Technologies, Inc. PDI is a single particle counter using a flux sampling technique. The physical principles underlying the PDI have been well documented in numerous publications, including Ref. 9. The Artium PDI system splits a laser beam and focuses the two resulting coherent beams to a common point in space, creating an interrogation volume and generating a local interference fringe pattern. Particles passing through this volume will scatter the light, creating a Doppler burst signal as they pass the interference fringe pattern. The Artium Technologies, Inc. PDI measures this Doppler burst with three detectors at separate spatial locations. The resulting phase shift of the Doppler burst signals allows measurement of the spacing of the interference fringe pattern, which is used to determine particle size. Using three detectors provides a means to avoid phase ambiguity when the phase cycles past $360^{\circ}$, and also provides redundant measurements that are used to validate the signals, offering an indication as to whether the particles are quasi-spherical or irregular-shaped ice particles. The system can size spherical and quasi-spherical particles. Irregularly shaped and partially melted particles will not follow the calibrated phase shift-size relationship, which provides an avenue for particle material phase and morphology discrimination.

The Artium Technologies, Inc. HSI is another particle sizing instrument that uses a spatial sampling technique. The HSI acquires high-resolution images of particles passing through the interrogation volume. This volume is created by focusing several laser beams on a common spatial point, illuminating particles for image capture by a CMOS camera with a long range Schmidt-Cassegrain telescope, which is recording at $300 \mathrm{~Hz}$. The lasers are simultaneously pulsed with a pulse duration on the nanosecond time-scale, reducing exposure times and motion blur of the images. With knowledge of the system resolution determined during setup calibration, the system can size spherical and irregularly shaped particles, and quantitative assessments of particle morphology can be made, allowing for potential identification of glaciated particles. The resolution for the HSI during the test was $1.8 \mu \mathrm{m} / \mathrm{pixel}$.

The limitations of any drop sizing instrument should be understood when comparing systems. Thus, it should be noted that the HSI has a larger minimum measurable particle size than the PDI, which can measure particles below 1 $\mu \mathrm{m}$, because a single darkened pixel is insufficient to identify and measure a particle in an image. Additionally, the size of the sample volume for the HSI is, in general, smaller than the PDI, and decreases in relative size to the PDI sample volume with increasing airspeed. This is because the sample volume size for the HSI is independent of the airspeed, while the sample volume size for the PDI is proportional to the airspeed. 
The PDI and HSI used during the test were modular, and the non-intrusive nature of the instrumentation allowed the systems to be used across several test days without interfering with other measurements and studies. Figure 1 shows the experimental setup for the NACA 0012 ice accretion studies, which was used for 2 of the 8 testing days. The PDI and HSI, which are labeled in Fig. 1, were focused approximately $152 \mathrm{~mm}$ above the centerline for these days. The PDI and HSI were also used during the test day dedicated to the DMT CDP and CIP-GS particle measurements, but only during the CDP portion of testing. The PDI and HSI were focused approximately $152 \mathrm{~mm}$ above the centerline for this portion of the test as well. More information on the positioning of the instrumentation and the test article during the test is available in Ref. 7. Unfortunately, no measurements were made at the centerline of the duct with the PDI or HSI during the first Fundamentals test.

\section{B. Select Cases}

Table 1 outlines the select cases examined in this paper. The cases are separated into two series. The first series is a humidity sweep of the PSL plenum conditions at a large $d_{v 0.50}, 50 \mu \mathrm{m}$, based on the NASA Icing Research Tunnel (IRT) drop size calibration, and the second series is a humidity sweep at a small $d_{v 0.50}, 15 \mu \mathrm{m}$, also based on the IRT drop size calibration. Varying the plenum humidity varies the local wet bulb temperature, $T_{W B}$. The wet bulb temperature affects the freeze-out of the cloud particles in the plenum, which is where the cloud is expected to have the greatest residence time ${ }^{10}$. The test points in Table 1 are arranged in order of decreasing $T_{W B}$.

The station 1 total water content, $T W C_{S l}$, values defined in Table 1 is are bulk averaged values, which assume that the cloud is uniformly distributed within the innermost $610 \mathrm{~mm}$ diameter of the station 1 plane. The spray bar nozzle pattern was optimized to generate a cloud that did not extend outside the innermost $610 \mathrm{~mm}$ diameter at station 1 . Refer to Ref. 7 for more detail on the station 1 total water content and cloud uniformity.

Table 1. Select Test Points

\begin{tabular}{|c|c|c|c|c|c|c|c|}
\hline Test Point & $p_{P L}$ & $\phi_{P L}$ & $T_{W B}{ }^{*}$ & $u_{S I}$ & $M_{S 1}$ & $T W C_{S I}$ & $d_{v 0.50^{\dagger}}$ \\
\hline--- & psi & $\%$ & ${ }^{\circ} \mathrm{C}$ & $\mathrm{m} / \mathrm{s}$ & --- & $\mathrm{g} / \mathrm{m}^{3}$ & $M m$ \\
\hline \multicolumn{8}{|c|}{ Large $d_{v 0.50}$ Series } \\
\hline 210 & 6.5 & 60 & 2.4 & 85 & 0.25 & 2 & 50 \\
\hline 209 & 6.5 & 47 & 0.6 & 85 & 0.25 & 2 & 50 \\
\hline 206 & 6.5 & 40 & -0.4 & 85 & 0.25 & 2 & 50 \\
\hline 204 & 6.5 & 25 & -2.6 & 85 & 0.25 & 2 & 50 \\
\hline 202 & 6.5 & 20 & -3.3 & 85 & 0.25 & 2 & 50 \\
\hline 201 & 6.5 & 10 & -4.9 & 85 & 0.25 & 2 & 50 \\
\hline 201.1 & 6.5 & 0 & -6.0 & 85 & 0.25 & 2 & 50 \\
\hline \multicolumn{8}{|c|}{ Small $d_{v 0.50}$ Series } \\
\hline 211 & 6.5 & 60 & 2.4 & 85 & 0.25 & 2 & 15 \\
\hline 208 & 6.5 & 47 & 0.6 & 85 & 0.25 & 2 & 15 \\
\hline 207 & 6.5 & 40 & -0.4 & 85 & 0.25 & 2 & 15 \\
\hline 207.1 & 6.5 & 38 & -0.5 & 85 & 0.25 & 2 & 15 \\
\hline 205 & 6.5 & 25 & -2.6 & 85 & 0.25 & 2 & 15 \\
\hline 203 & 6.5 & 20 & -3.3 & 85 & 0.25 & 2 & 15 \\
\hline
\end{tabular}

${ }^{*}$ Values based on conditions in the plenum

$\dagger$ Values based on NASA Icing Research Tunnel cloud drop size calibration and do not represent

PDI or HSI test data

\section{Results and Discussion}

Figures referenced in this section are located at the end of the paper. The figures are arranged in the order of decreasing $T_{W B}$ as shown in Table 1 for each series, and each test point figure contains five subfigures, including (a) cumulative percent volume distribution comparison, (b) number density comparison, (c) water content comparison, (d) area-perimeter correlation, and (e) raw signal phase difference plots. While the first three plots provide useful comparison between the PDI and HSI, the area-perimeter and raw signal phase difference plots provide an indication of the morphology of the particles, which is useful when analyzing the results. The area-perimeter plots are developed 
from the HSI data where spherical particles are expected to closely follow the theoretical line for a circle, $A R=1$ line. The $A R=2$ and $A R=3$ represent ellipses with major diameters 2 and 3 times the unitary minor diameter, respectively, and are intended to provide frames of reference. Similarly, the raw signal phase difference plots are developed from the PDI data where spherical particles are expected to closely follow the dashed lines, as described in the Experimental Description section.

\section{A. Large $d_{v 0.50}$ Series}

The PDI and HSI particle size distributions (PSD) agree reasonably well throughout the Large $d_{v 0.50}$ Series, which can be seen in Fig. 2 through 8. Examining Table 2, which shows normalized cumulative percent volume distribution values, the PSDs for both instruments appear independent of the decreasing $T_{W B}$ in the series. The number densities and water content distributions for both instruments also agree throughout the series, but appear low, which may indicate that the PDI and HSI were measuring close to the outside perimeter of the cloud. It is clear from the number density and water content comparisons that the HSI rolls off towards the lower end of the particle spectrum faster than the PDI. However, this behavior is expected because the HSI has a larger minimum measurable particle size than the PDI. At the other end of the spectrum, the HSI cuts off sooner that the PDI. Again, this behavior is expected because of difference in the sample volume size between two instruments. Throughout the rest of the distribution, the PDI and HSI agree well.

Progressively examining each test point in the series reveals a gradual change in the raw signal phase difference plots from the PDI and the particle area-perimeter correlation from the HSI. As the $T_{W B}$ decreases, the scatter in the raw signal phase difference plots increases, and the data trends away from the theoretical line for a circle $(A R=1)$ in the area-perimeter correlation, both indicating increasing presense of irregularly shaped ice particles. The sample particle images in Fig. 9 and 10 show the morphology difference between test points 210 and 201.1 supporting this indication. These items demonstrate the current ability to assess particle morphology using the PDI and HSI, and a future potential avenue for statistical morphology characterization of ICI conditions. The particles lying further from theoretical lines in both the raw signal phase plots and the area-perimeter correlation plots are believed to be glaciated, but further validation is required to corroborate such assessments.

Table 2. Large $d_{v 0.50}$ Series Normalized Cumulative Volume Distributions

\begin{tabular}{cccccccc}
\hline Test Point & $d_{\min }$ & $d_{v 0.10}$ & $d_{v 0.25}$ & $d_{v 0.50}$ & $d_{v 0.90}$ & $d_{v 0.99}$ & $d_{\max }$ \\
\hline--- & $\mu m$ & $\mu m$ & $\mu m$ & $\mu m$ & $\mu m$ & $M m$ & $\mu m$ \\
PDI & & & & & & & \\
\hline 210 & 6 & 26 & 37 & 53 & 97 & 137 & 164 \\
209 & 7 & 27 & 37 & 53 & 97 & 138 & 156 \\
206 & 5 & 27 & 37 & 53 & 96 & 139 & 155 \\
204 & 5 & 27 & 37 & 52 & 97 & 143 & 177 \\
202 & 4 & 27 & 37 & 53 & 97 & 130 & 141 \\
201 & 1 & 25 & 36 & 52 & 92 & 141 & 149 \\
201.1 & 1 & 26 & 36 & 52 & 94 & 139 & 143 \\
$H S I$ & & & & & & & \\
\hline 210 & 6 & 24 & 33 & 47 & 98 & 129 & 137 \\
209 & 7 & 25 & 32 & 47 & 88 & 124 & 125 \\
206 & 5 & 25 & 33 & 47 & 89 & 159 & 160 \\
204 & 5 & 26 & 35 & 49 & 86 & 119 & 125 \\
202 & 4 & 26 & 36 & 50 & 90 & 116 & 130 \\
201 & 1 & 27 & 38 & 57 & 99 & 116 & 118 \\
201.1 & 1 & 28 & 37 & 52 & 87 & 117 & 123 \\
\hline
\end{tabular}

\section{B. Small $d_{v 0.50}$ Series}

Inspecting Fig. 11 through 16, it is apparent the PDI and HSI PSD do not agree well in the Small $d_{v 0.50}$ Series. Acquiring the PSD with the HSI is difficult because the distribution is concentrated towards the lower end of the size spectrum, and the roll off of the HSI towards the lower end is more apparent in the Small $d_{v 0.50}$ Series number density and water content comparisons than the Large $d_{v 0.50}$ Series comparisons. Table 3 shows decreasing independence of 
the PSD for the PDI to decreasing $T_{W B}$, while the HSI PSD remains consistent, but shifted towards the higher end of size spectrum, throughout the series. This indicates that the PSD is not changing, but rather, the particles are becoming more irregularly shaped, reducing validation on the lower end of the particle spectrum for the PDI. These items indicate that further development of the PDI and HSI towards measuring the lower end of the irregular-shape particle spectrum is necessary.

Again, each test point in the series reveals a gradual increase in the scatter in the raw signal phase difference plots from the PDI, similar to that observed in the Large $d_{v 0.50}$ Series. The data trending away from the $A R=1$ line in the area-perimeter correlation plots from the HSI is evident upon inspection, but a bit easier to distinguish than the Large $d_{v 0.50}$ Series. Finally, the sample particle images in Fig. 17 and 18 show the morphology difference between test points 211 and 203, respectively. Thus, similar to the Large $d_{v 0.50}$ Series, the Small $d_{v 0.50}$ Series particle images show a clear increase in irregular-shape particle morphology with decreasing $T_{W B}$, indicating the presence of particles believed to be glaciated. Again, further validation is required to corroborate such assessments.

Table 3. Small $d_{v 0.50}$ Series Normalized Cumulative Volume Distributions

\begin{tabular}{cccccccc}
\hline Test Point & $d_{\min }$ & $d_{v 0.10}$ & $d_{v 0.25}$ & $d_{v 0.50}$ & $d_{v 0.90}$ & $d_{v 0.99}$ & $d_{\max }$ \\
\hline--- & $\mu m$ & $\mu m$ & $\mu m$ & $\mu m$ & $\mu m$ & $\mu m$ & $\mu m$ \\
PDI & & & & & & & \\
\hline 211 & 0.7 & 12 & 15 & 20 & 33 & 44 & 56 \\
208 & 0.6 & 11 & 15 & 20 & 33 & 47 & 56 \\
207 & 0.6 & 11 & 15 & 20 & 33 & 48 & 58 \\
207.1 & 0.5 & 12 & 16 & 22 & 43 & 65 & 82 \\
205 & 0.5 & 13 & 18 & 25 & 51 & 84 & 89 \\
203 & 0.5 & 13 & 18 & 26 & 54 & 83 & 87 \\
$H S I$ & & & & & & & \\
\hline 211 & 16 & 21 & 25 & 32 & 42 & 45 & 46 \\
208 & 12 & 20 & 23 & 29 & 42 & 55 & 62 \\
207 & 11 & 19 & 23 & 28 & 41 & 55 & 62 \\
207.1 & 11 & 19 & 23 & 28 & 40 & 50 & 62 \\
205 & 11 & 19 & 23 & 28 & 41 & 50 & 55 \\
203 & 9 & 19 & 23 & 29 & 41 & 67 & 68 \\
\hline
\end{tabular}

\section{Conclusions}

NASA has completed the first Fundamentals of Ice Crystal Icing Physics test in PSL. The Artium Technologies, Inc. PDI and HSI instruments were successfully used to measure the icing cloud during the test. The PDI and HSI demonstrated good agreement during the Large $d_{v 0.50}$ Series through a range of $T_{W B}$. Due to minimum measurable range of the HSI, there was not good agreement between the PDI and HSI during the Small $d_{v 0.50}$ Series. Thus, further development of the PDI and HSI towards measuring the lower end of the irregular-shape particle spectrum is necessary. The PDI raw signal phase difference and HSI area-perimeter correlation plots demonstrated the current capability for the instruments to allow researchers to examine particle morphology, and consequently discriminate glaciated conditions. Further validation is required, but initial analyses appear to indicate a potential avenue for a future capability to statisitically characterize ice crystal cloud material phase with the PDI and HSI. Thus, the results from the test offer insight into the current capabilities of the PDI and HSI, and a representative data set of ICI conditions to drive future development towards improved measurement and characterization of hazardous icing conditions.

\section{Acknowledgments}

The authors wish to acknowledge the financial support for this work by the Advanced Aircraft Icing (AAI) Subproject of the NASA Advanced Air Transport Technology Project (AATT) under NASA's Advanced Air Vehicles program. 


\section{References}

${ }^{1}$ Mason, J. G., Strapp, J. W., and Tsao, J. C., “The Ice Particle Threat to Engines in Flight," 44th AIAA Aerospace Sciences Meeting and Exhibit, AIAA-2006-206, 2006.

${ }^{2}$ Oliver, M. J., "Validation Ice Crystal Icing Engine Test in the Propulsion Systems Laboratory at NASA Glenn Research Center," 6th AIAA Atmospheric and Space Environments Conference, AIAA-2014-2898, 2014.

${ }^{3}$ Goodwin, R. V., and Dischinger, D. G., "Turbofan Ice Crystal Rollback Investigation and Preparations Leading to Inaugural Ice Crystal Engine Test at NASA PSL-3 Facility,” 6th AIAA Atmospheric and Space Environments Conference, AIAA-2014-2895, 2014.

${ }^{4}$ Tsao, J. C., Struk, P. M., and Oliver, M. J., "Possible Mechanisms for Turbofan Engine Ice Crystal Icing at High Altitude," 6th AIAA Atmospheric and Space Environments Conference, AIAA 2014-3044, 2014.

${ }^{5}$ Flegel, A. B., Oliver, M. J., "Preliminary Results from a Heavily Instrumented Engine Ice Crystal Icing Test in a Ground Based Altitude Test Facility," 8th AIAA Atmospheric and Space Environments Conference, AIAA 2016-3894, 2016.

${ }^{6}$ Struk, P. M., Taso, J. C., and Bartkus, T. P., "Plans and Preliminary Results of Fundamnental Studies of Ice Crystal Icing Physics in the NASA Propulsion Systems Laboratory," 8th AIAA Atmospheric and Space Environments Conference, AIAA-20163738, 2016.

${ }^{7}$ Struk, P. M., Ratvasky, T. P., Bencic, T. J., Van Zante, J. F., King, M. C., Taso, J. C., and Bartkus, T. P., “An Initial Study of the Fundamentals of Ice Crystal Icing Physics in the NASA Propulsion Systems Laboratory," 9th AIAA Atmospheric and Space Environments Conference, American Institute of Aeronautics and Astronautics, Denver, CO (submitted for publication).

${ }^{8}$ Van Zante, J. F., Bencic, T. J., Ratvasky, T. P., "NASA Glenn Propulsion Systems Lab Ice Crystal Cloud Characterization Update 2015," 8th AIAA Atmospheric and Space Environments Conference, AIAA-2016-3897, 2016.

${ }^{9}$ Albrecht, H.-E., Damaschke, N., Borys, M., and Tropea, C., Laser Doppler and Phase Doppler Measurement Techniques, Springer-Verlag, Berlin, 2003.

${ }^{10}$ Bartkus, T. P., Struk, P. M., and Tsao, J. C. "Comparisons of Mixed-Phase Icing Cloud Simulations with Experiments Conducted at the NASA Propulsion Systems Laboratory," 9th AIAA Atmospheric and Space Environments Conference, American Institute of Aeronautcs and Astronautics, Denver, CO (submitted for publication).

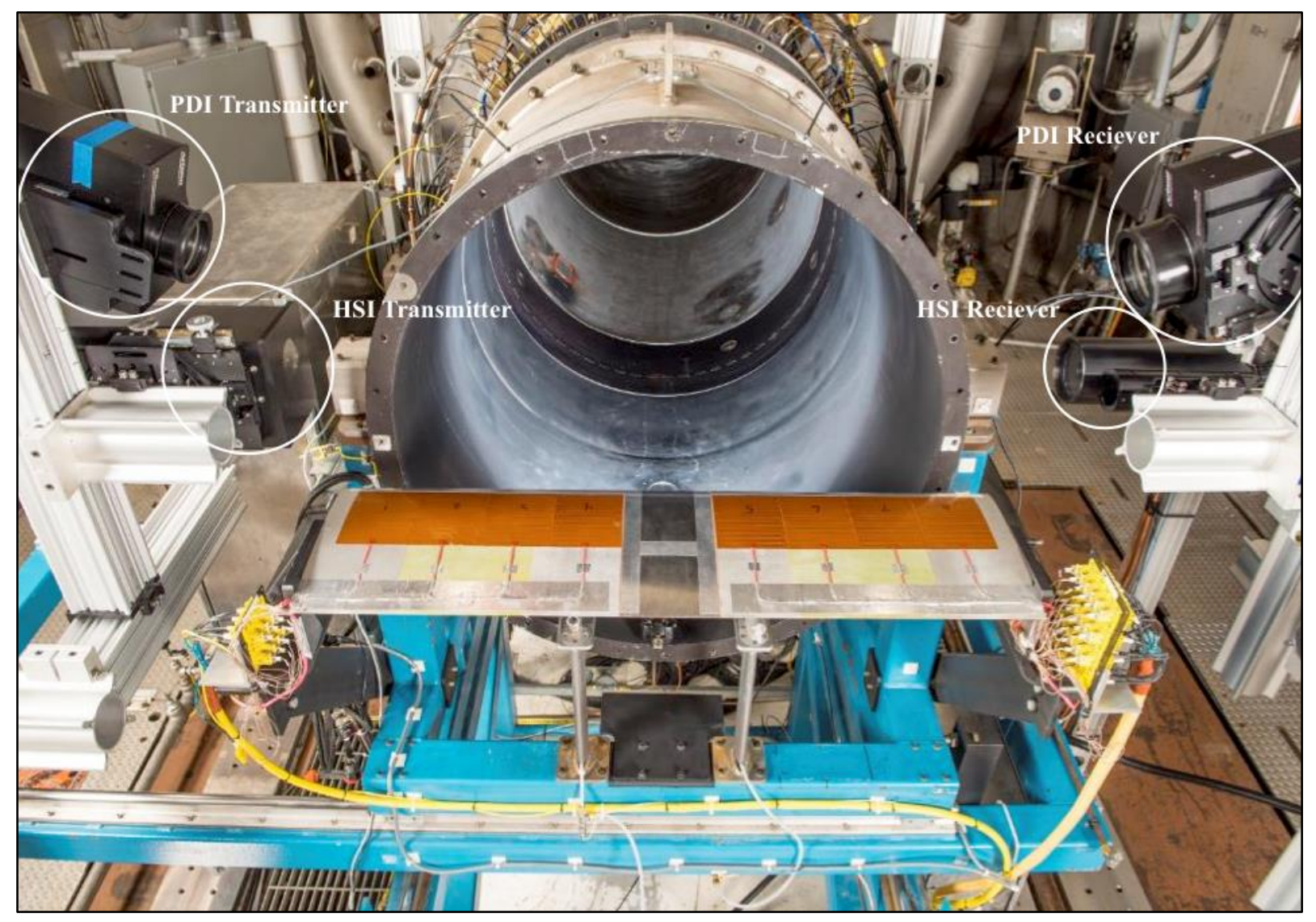

Figure 1. First Fundamental Ice Crystal Icing Test Configuration 2 setup, showing the Artium Technologies, Inc. Phase Doppler Interferometer and High Speed Imaging instruments focused approximately 152 millimeters above the duct centerline 


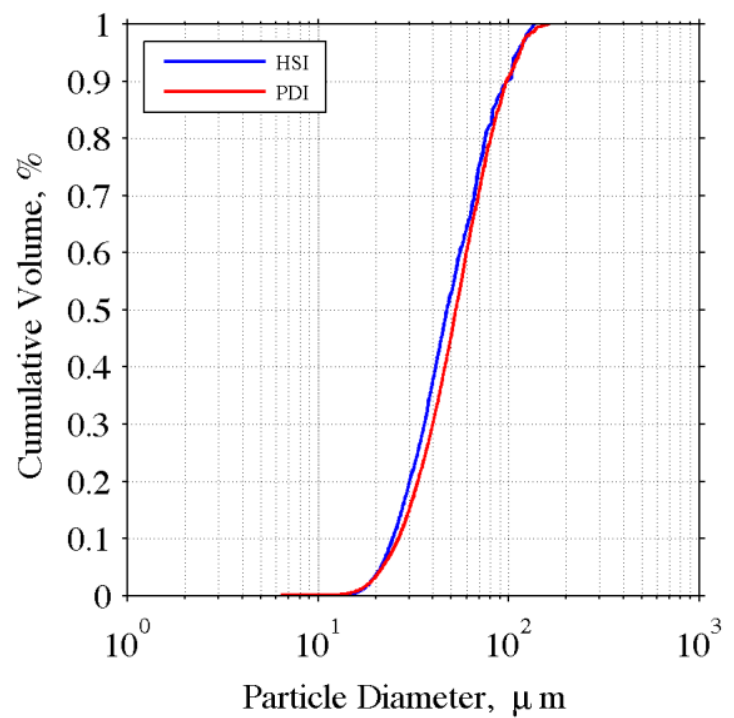

(a)

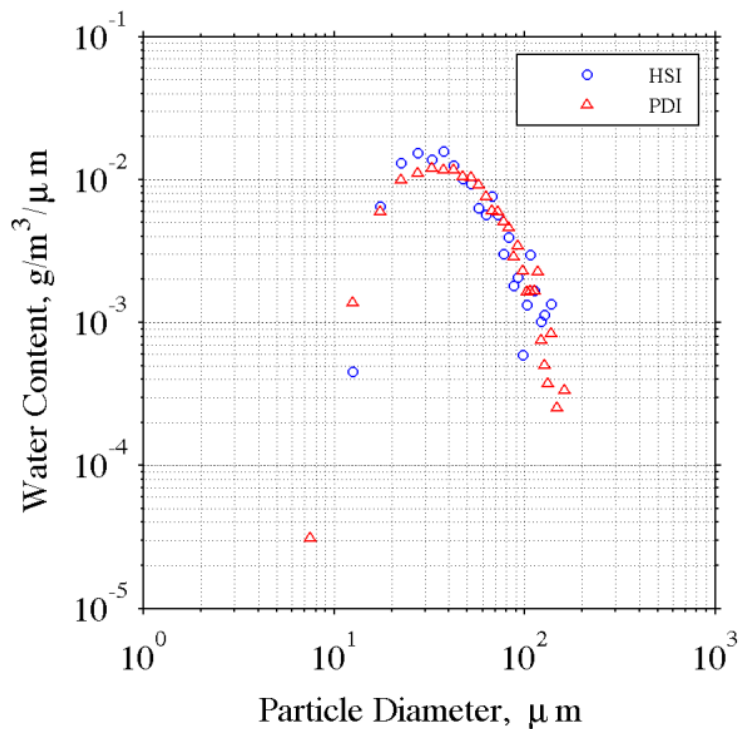

(c)

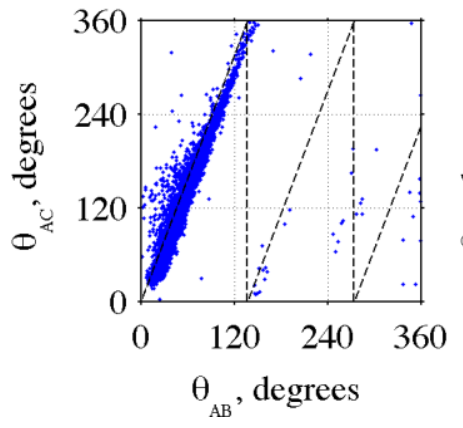

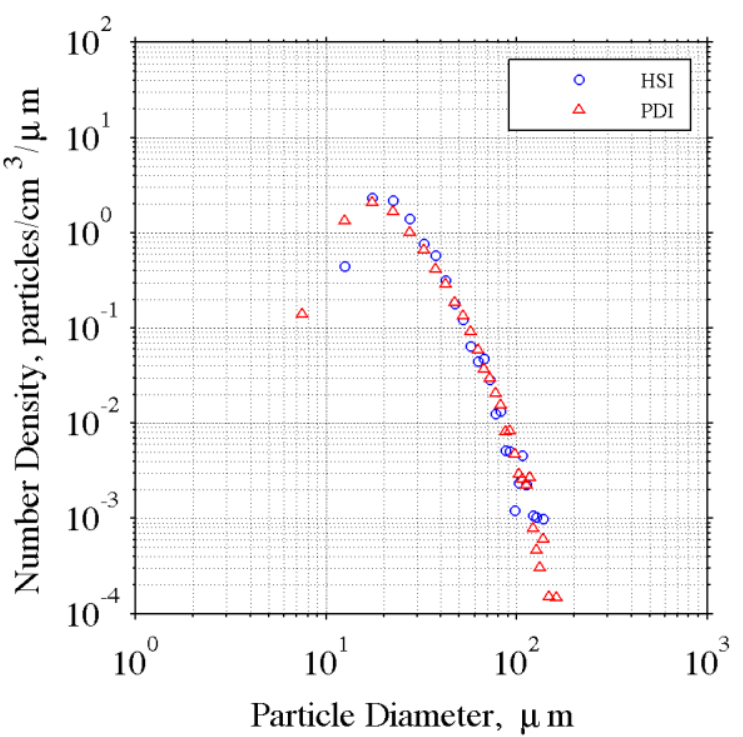

(b)

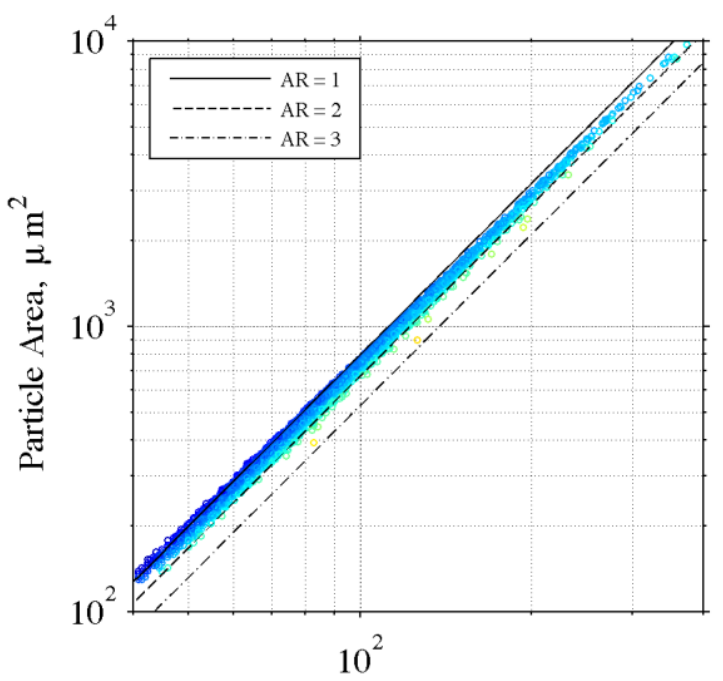

Particle Perimeter, $\mu \mathrm{m}$

(d)

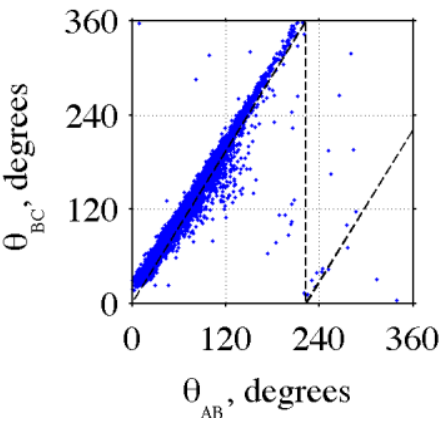

(e)

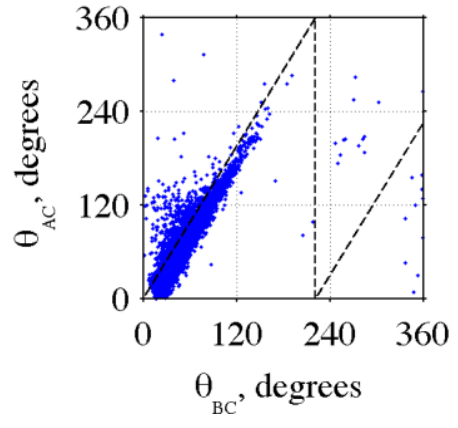

$\theta_{\mathrm{BC}}$, degrees

Figure 2. Test Point 210 Results showing (a) Cumulative Percent Volume Distribution PDI-HSI Comparison, (b) Number Density Distribution PDI-HSI Comparison, (c) Water Content Distribution PDI-HSI Comparison, (d) HSI Particle Area-Perimeter Correlation, and (e) PDI Raw Signal Phase Difference 


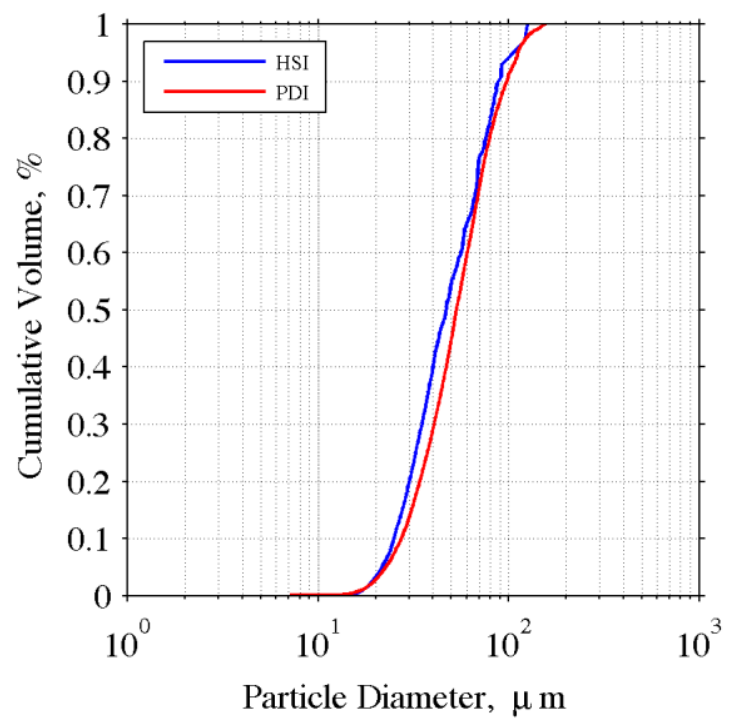

(a)

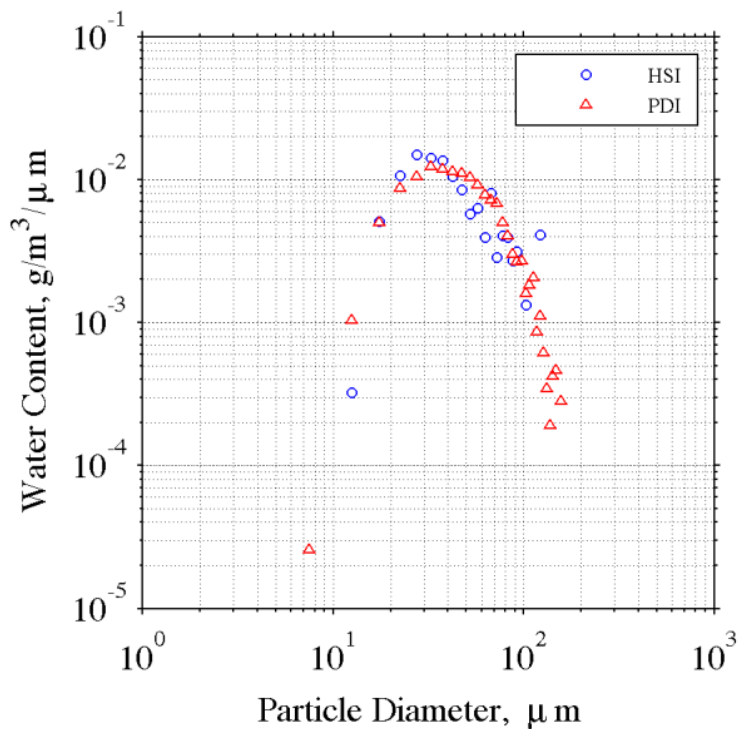

(c)

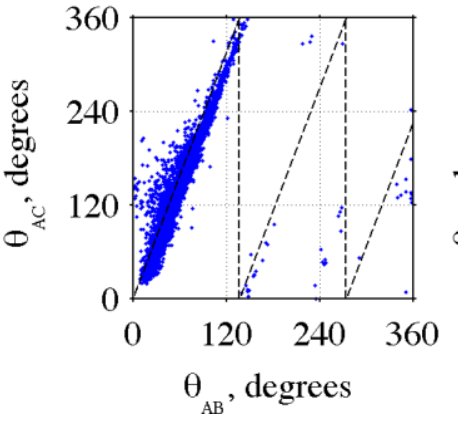

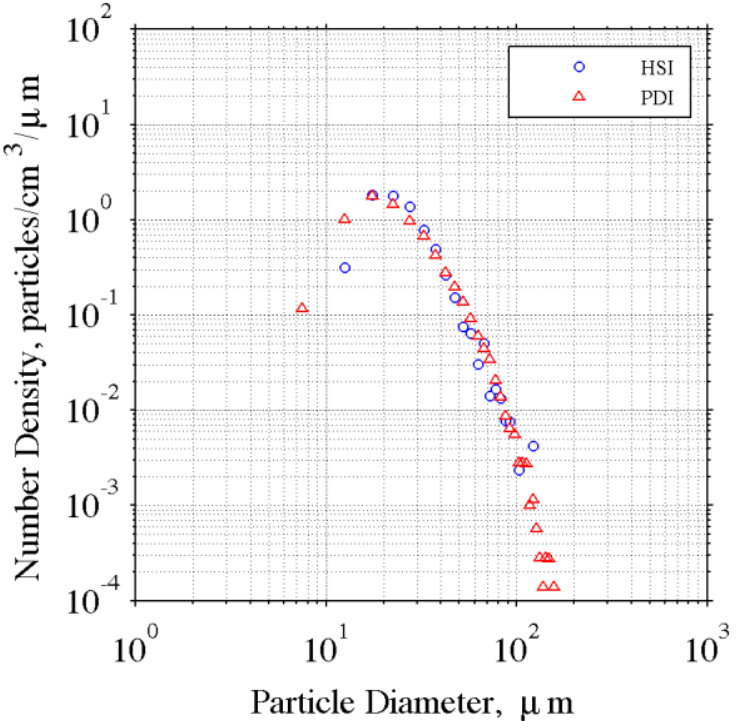

(b)

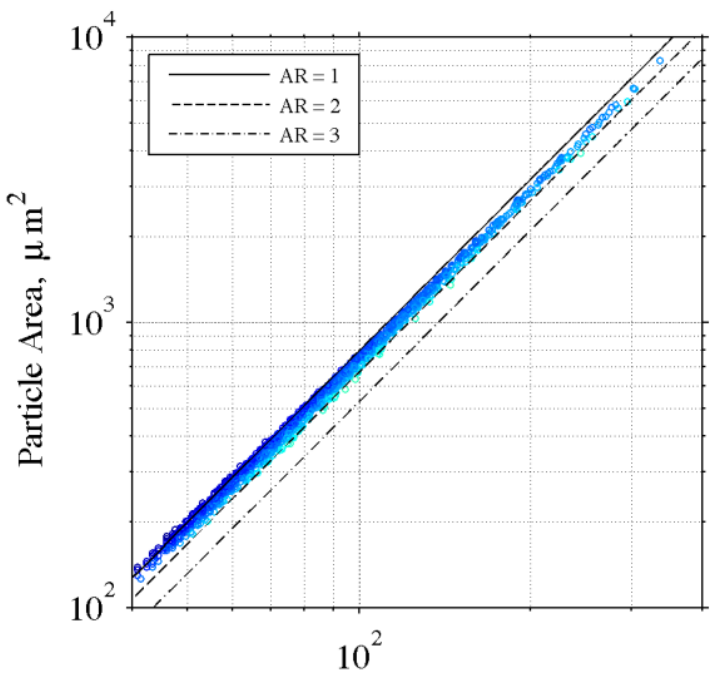

Particle Perimeter, $\mu \mathrm{m}$

(d)

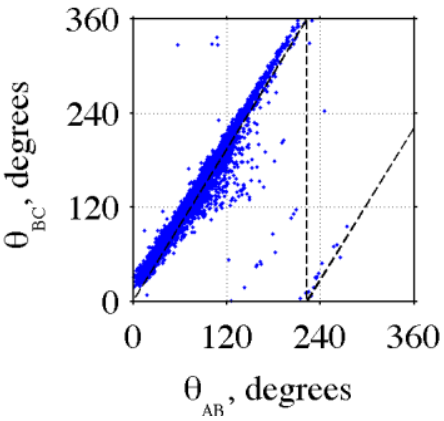

(e)

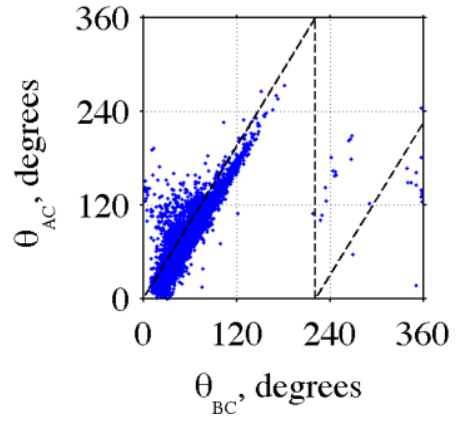

$\theta_{\mathrm{BC}}$, degrees

Figure 3. Test Point 209 Results showing (a) Cumulative Percent Volume Distribution PDI-HSI Comparison, (b) Number Density Distribution PDI-HSI Comparison, (c) Water Content Distribution PDI-HSI Comparison, (d) HSI Particle Area-Perimeter Correlation, and (e) PDI Raw Signal Phase Difference 


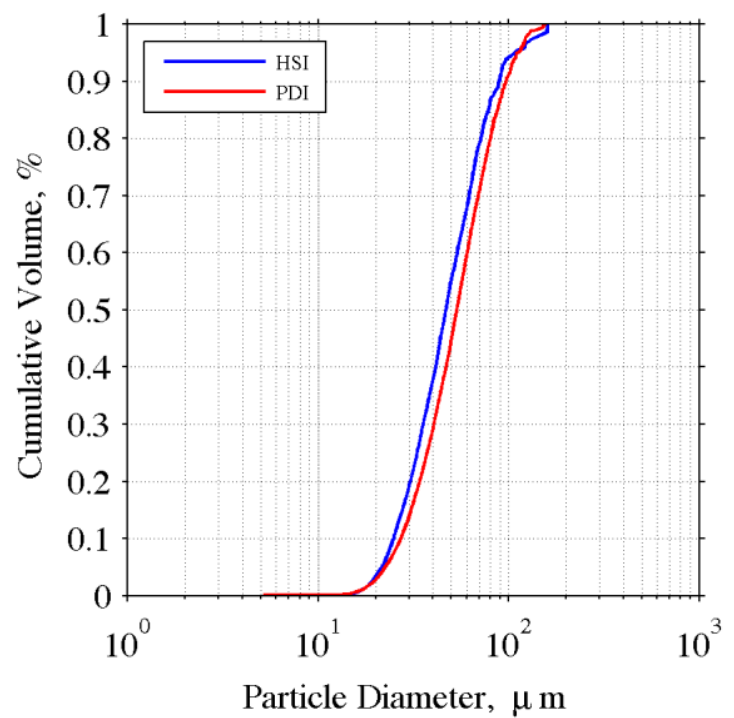

(a)

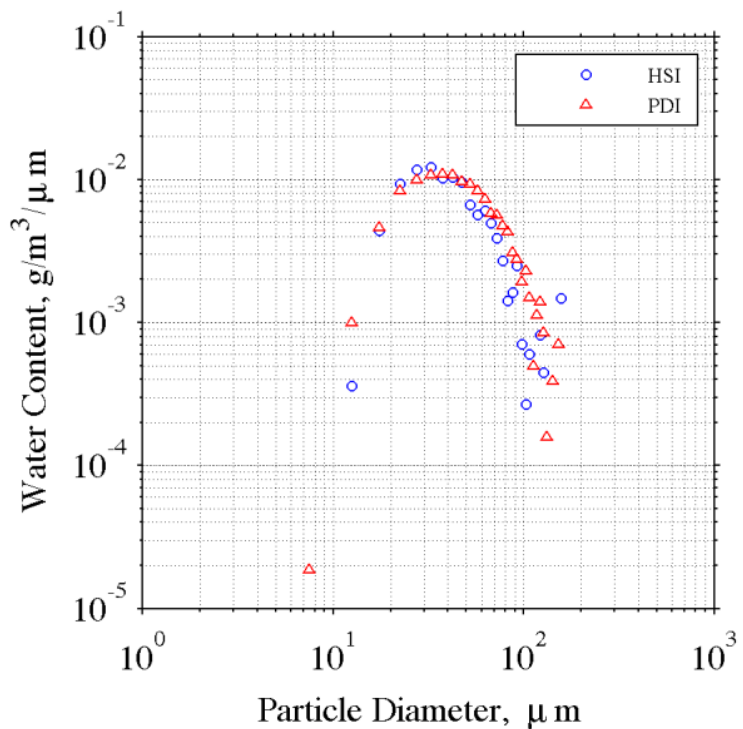

(c)

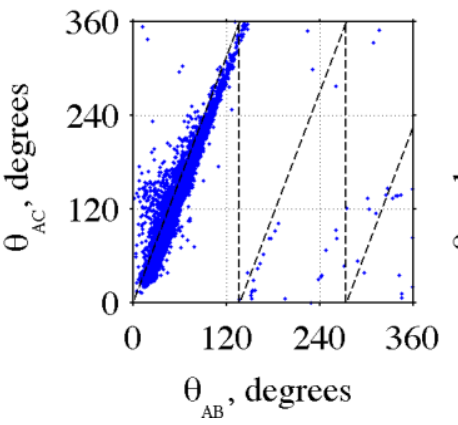

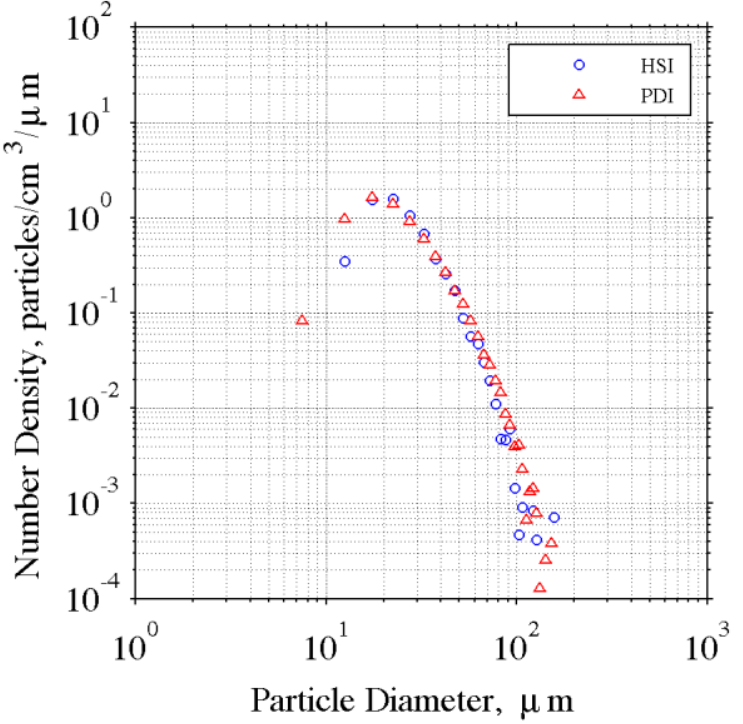

(b)

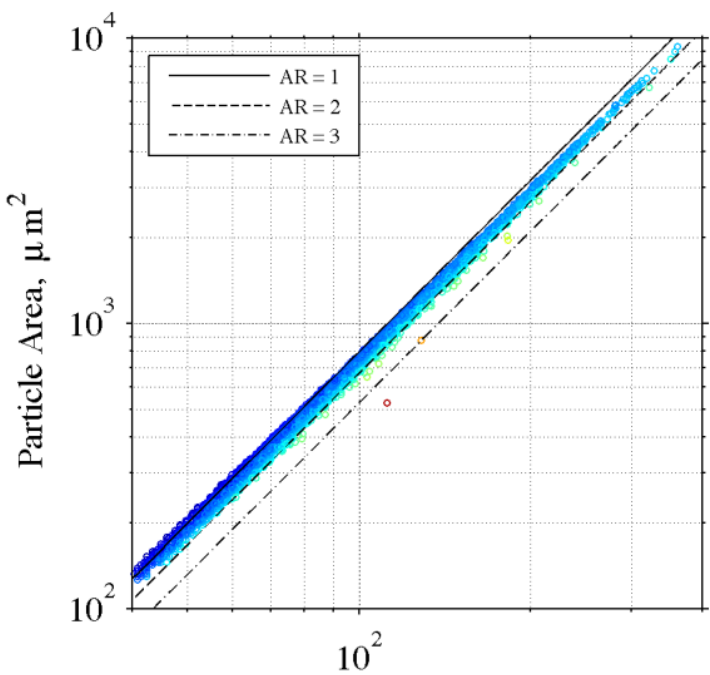

Particle Perimeter, $\mu \mathrm{m}$

(d)

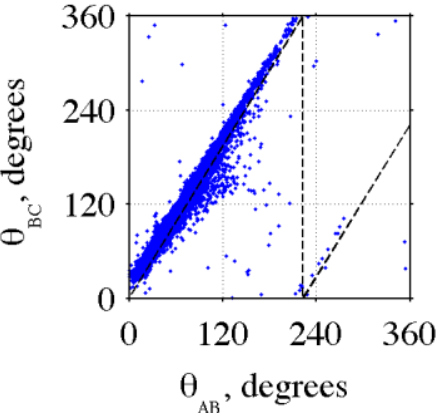

(e)

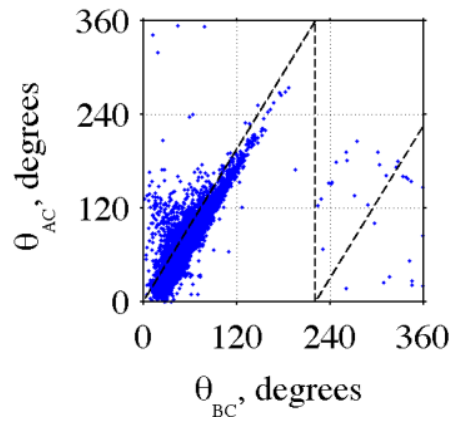

Figure 4. Test Point 206 Results showing (a) Cumulative Percent Volume Distribution PDI-HSI Comparison, (b) Number Density Distribution PDI-HSI Comparison, (c) Water Content Distribution PDI-HSI Comparison, (d) HSI Particle Area-Perimeter Correlation, and (e) PDI Raw Signal Phase Difference 


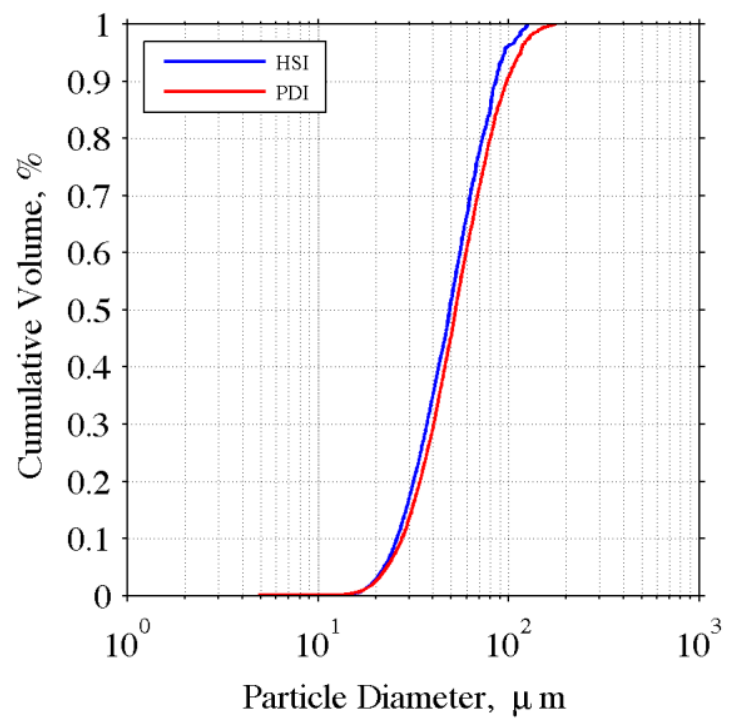

(a)

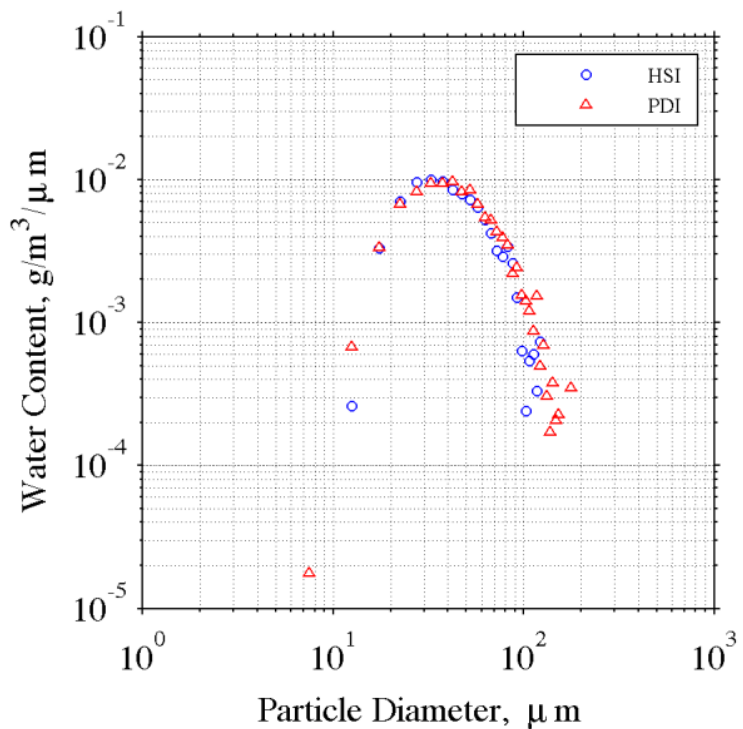

(c)

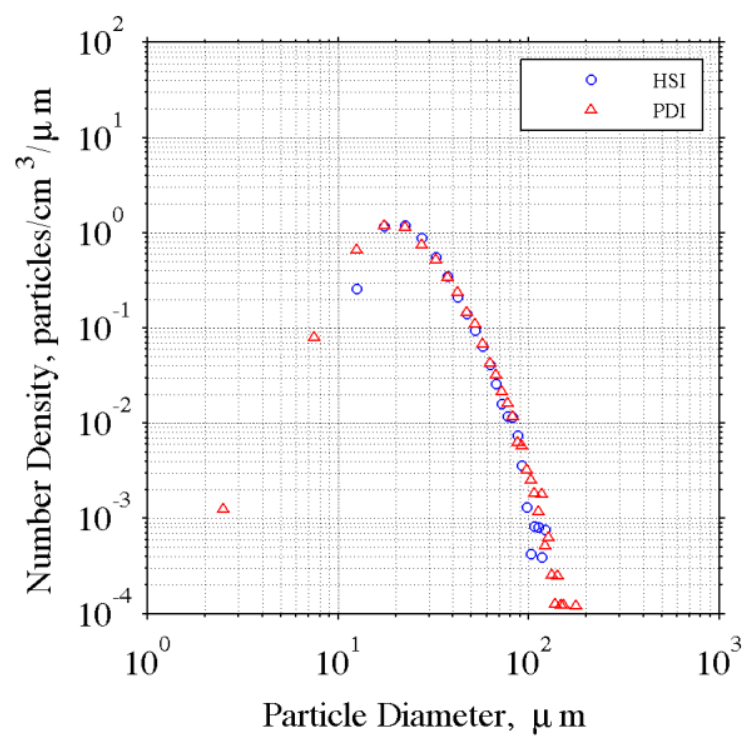

(b)

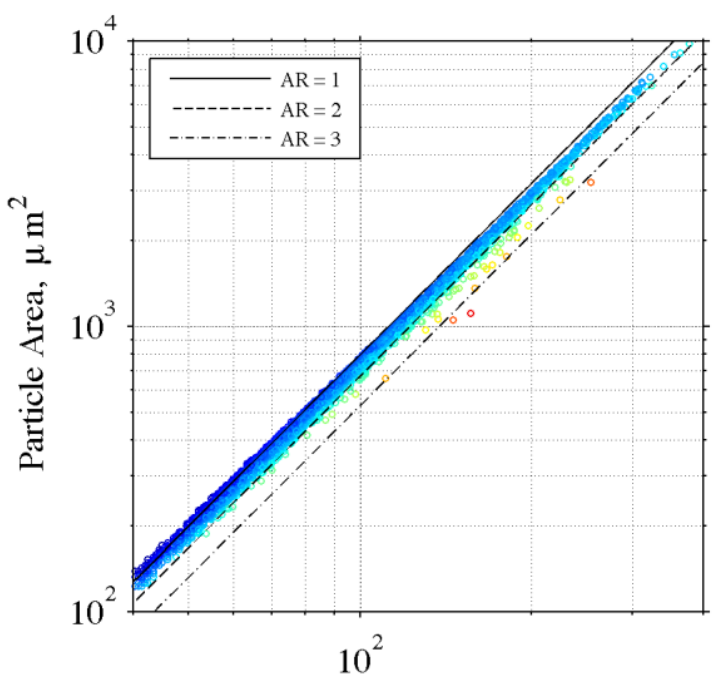

Particle Perimeter, $\mu \mathrm{m}$

(d)
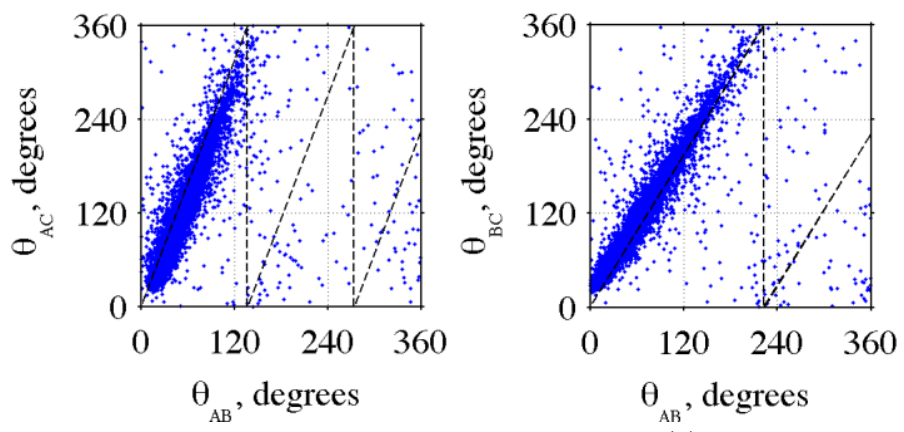

(e)

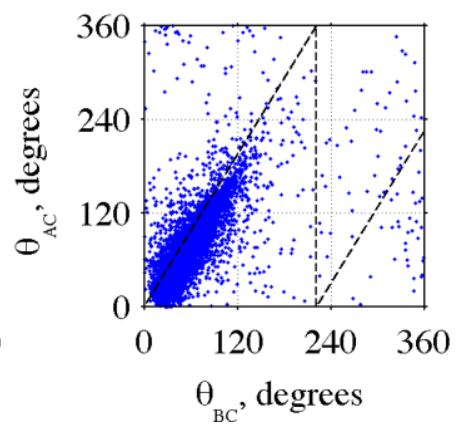

Figure 5. Test Point 204 Results showing (a) Cumulative Percent Volume Distribution PDI-HSI Comparison, (b) Number Density Distribution PDI-HSI Comparison, (c) Water Content Distribution PDI-HSI Comparison, (d) HSI Particle Area-Perimeter Correlation, and (e) PDI Raw Signal Phase Difference 


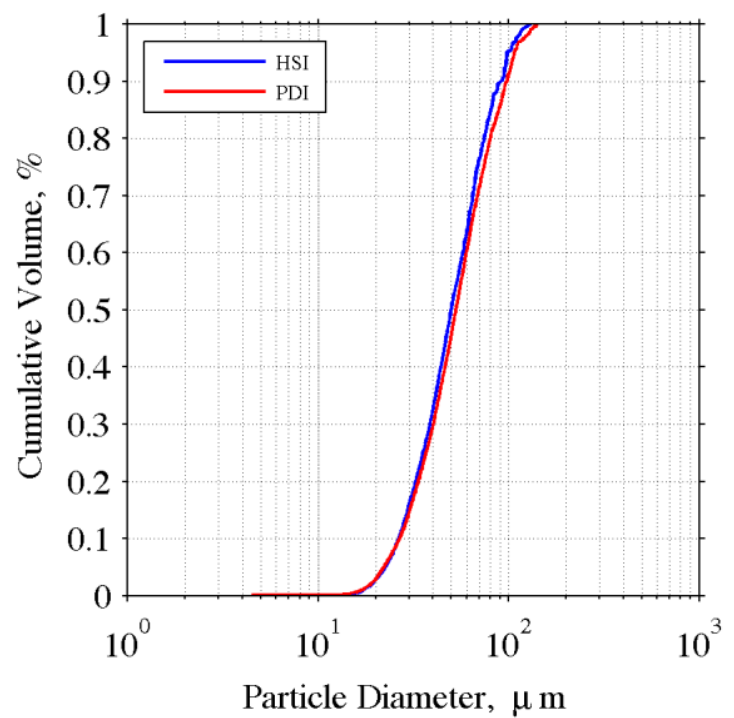

(a)

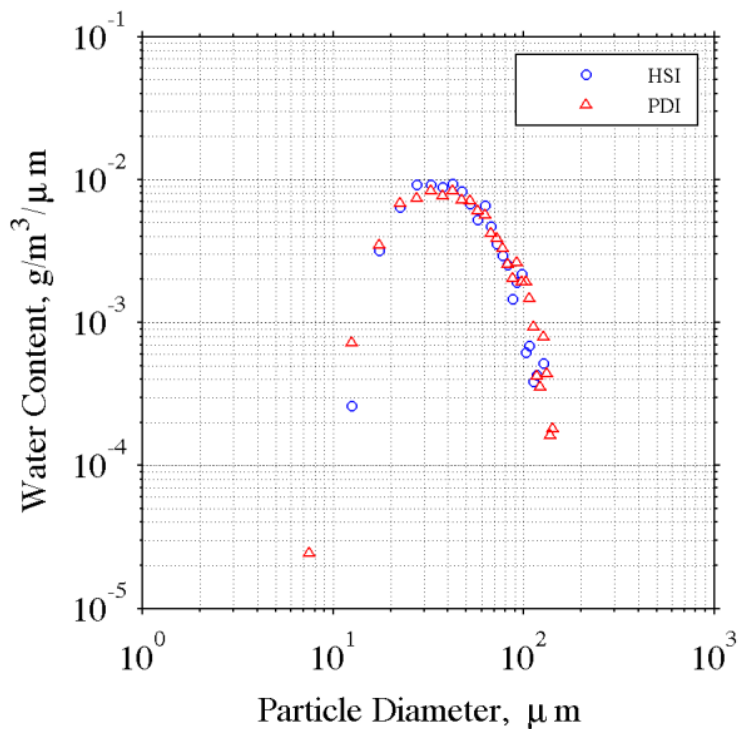

(c)

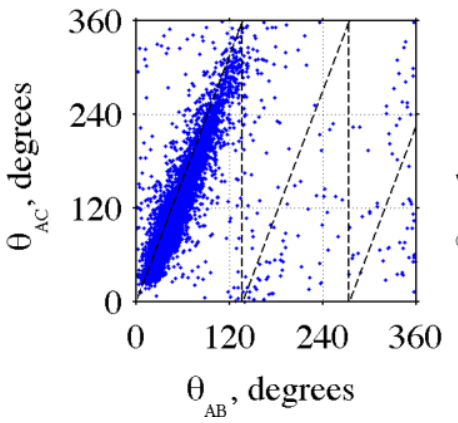

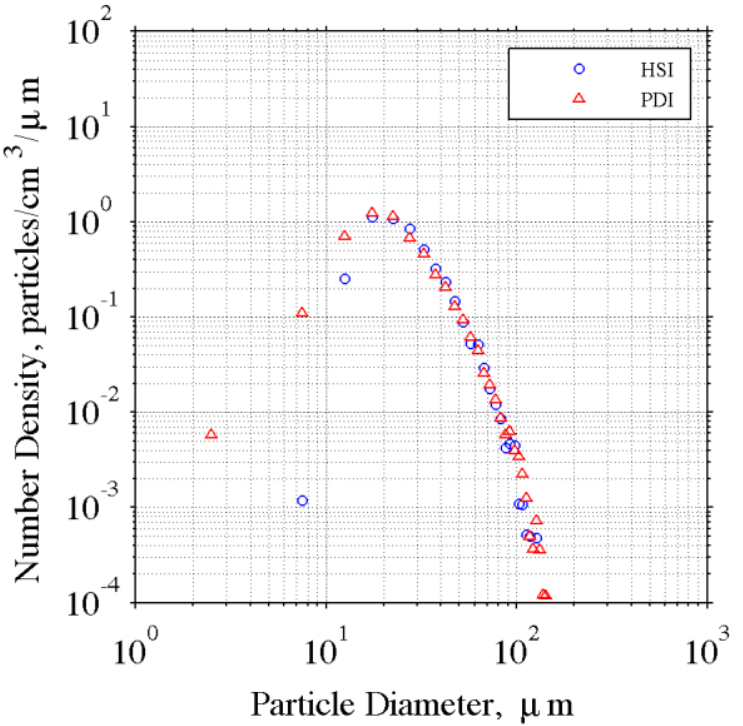

(b)

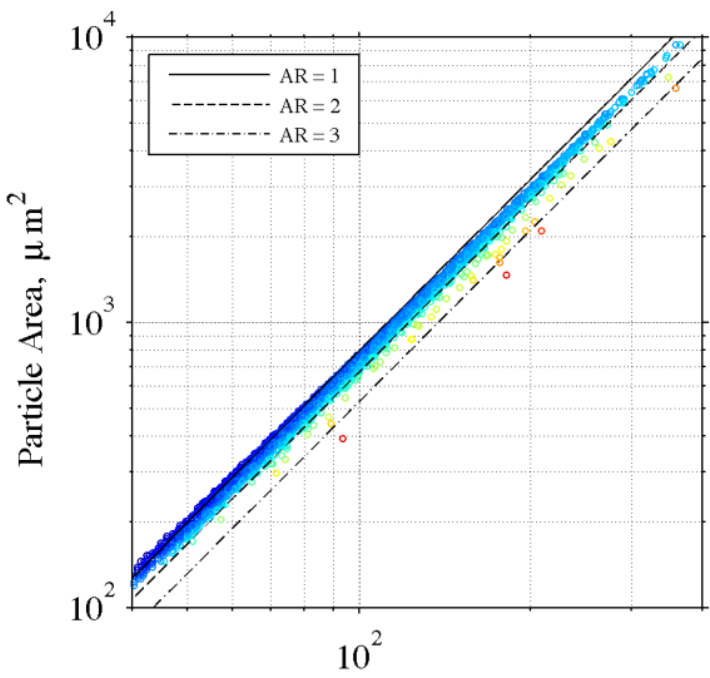

Particle Perimeter, $\mu \mathrm{m}$

(d)

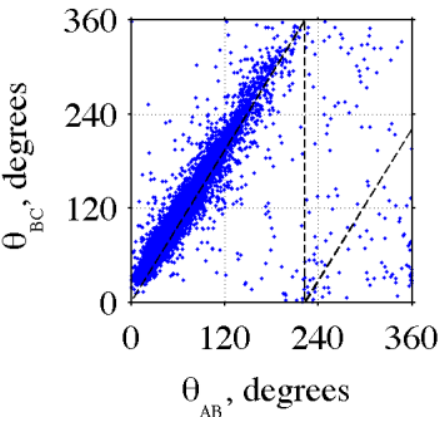

(e)

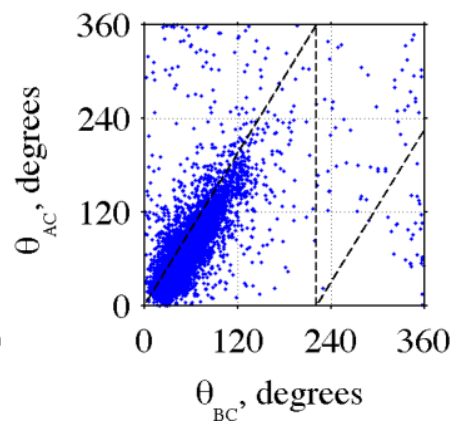

Figure 6. Test Point 202 Results showing (a) Cumulative Percent Volume Distribution PDI-HSI Comparison, (b) Number Density Distribution PDI-HSI Comparison, (c) Water Content Distribution PDI-HSI Comparison, (d) HSI Particle Area-Perimeter Correlation, and (e) PDI Raw Signal Phase Difference 


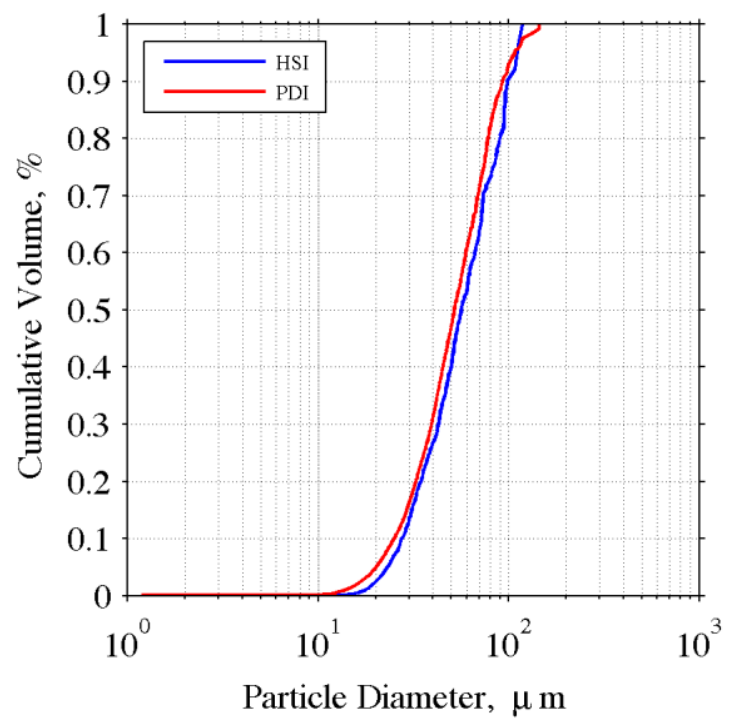

(a)

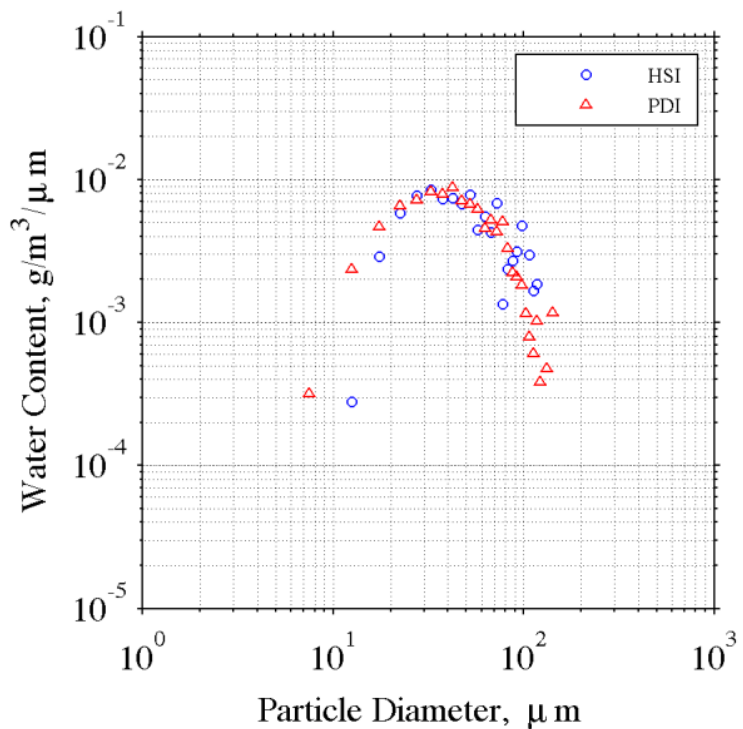

(c)

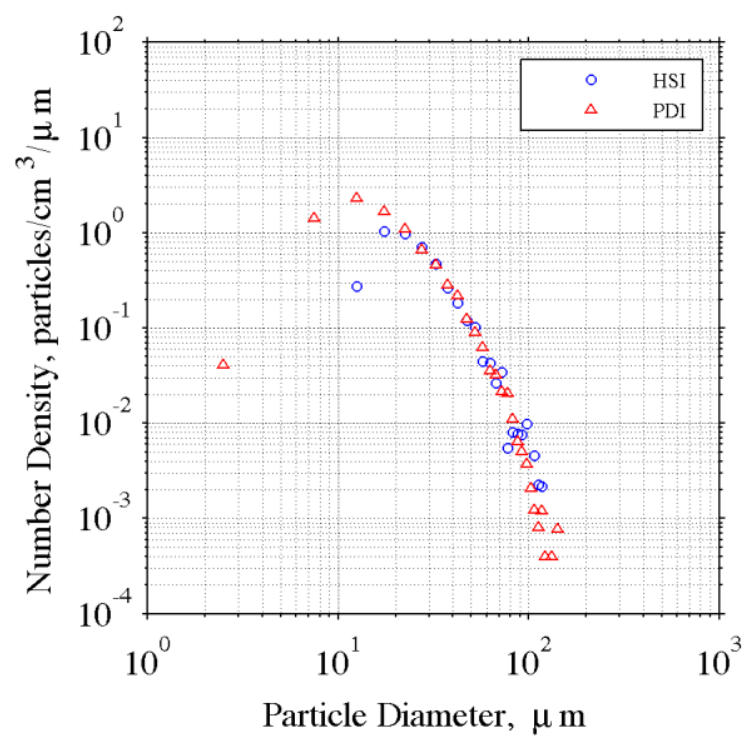

(b)

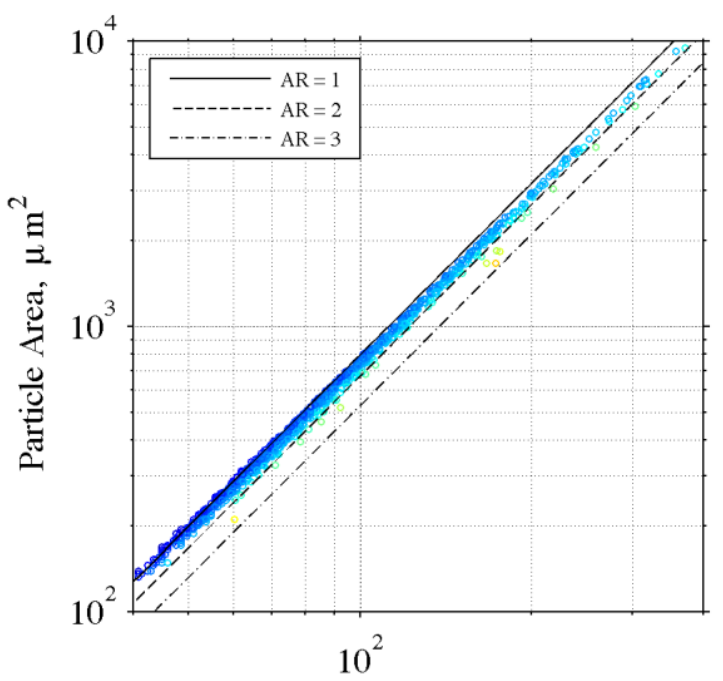

Particle Perimeter, $\mu \mathrm{m}$

(d)
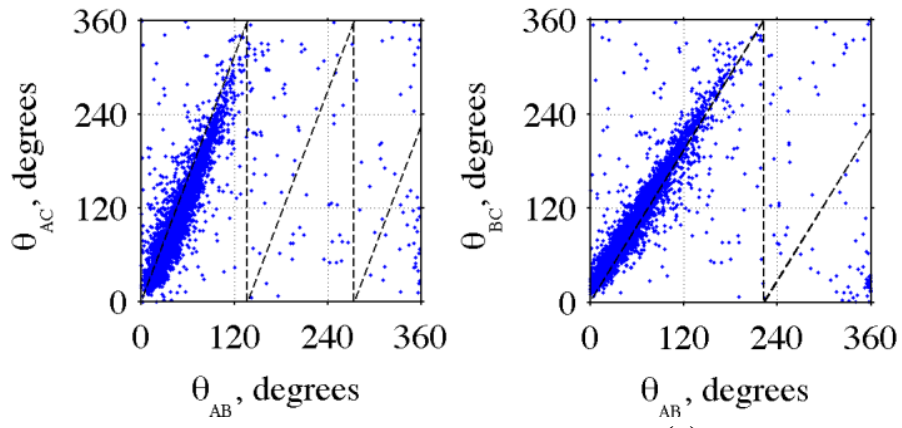

(e)

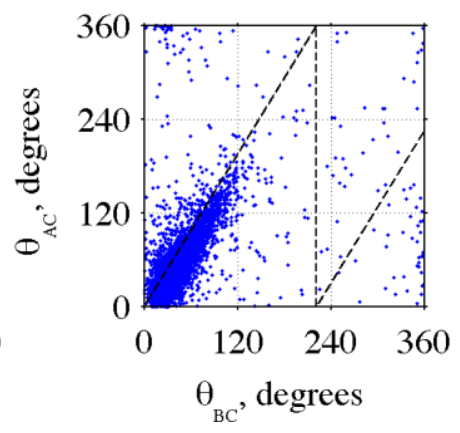

Figure 7. Test Point 201 Results showing (a) Cumulative Percent Volume Distribution PDI-HSI Comparison, (b) Number Density Distribution PDI-HSI Comparison, (c) Water Content Distribution PDI-HSI Comparison, (d) HSI Particle Area-Perimeter Correlation, and (e) PDI Raw Signal Phase Difference 


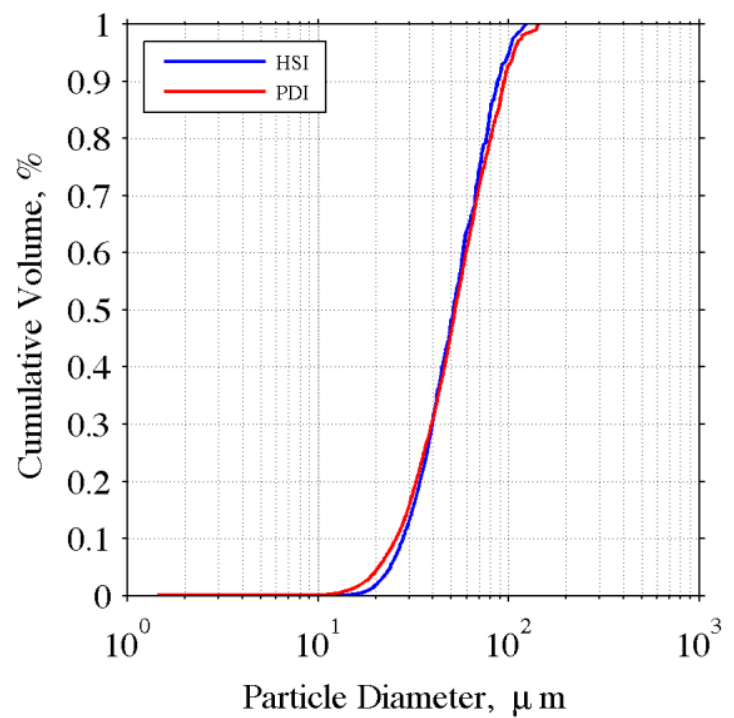

(a)

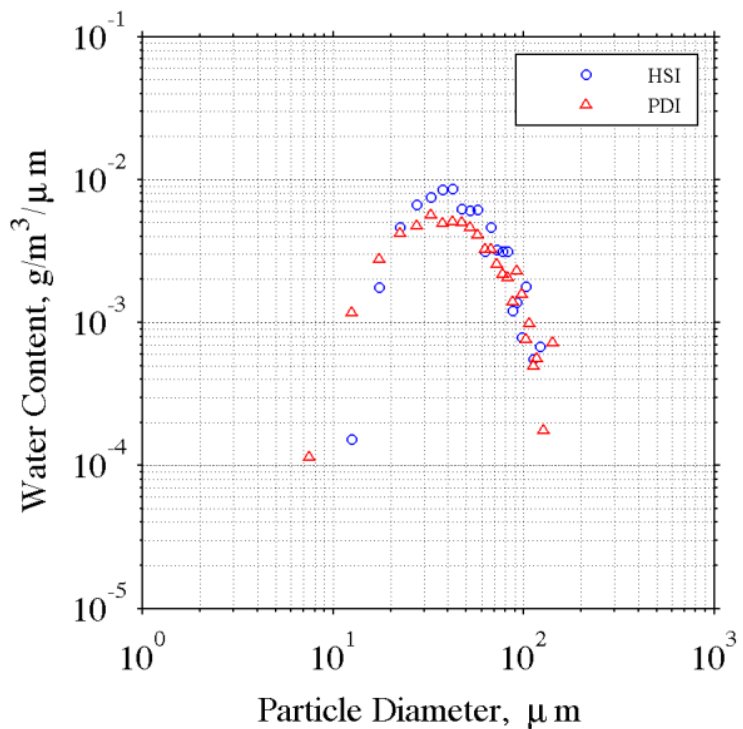

(c)

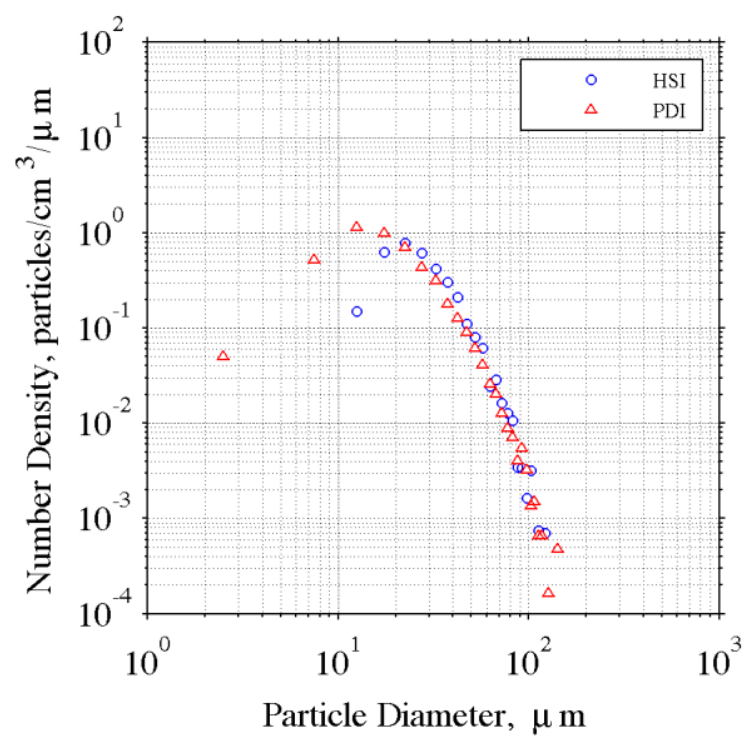

(b)

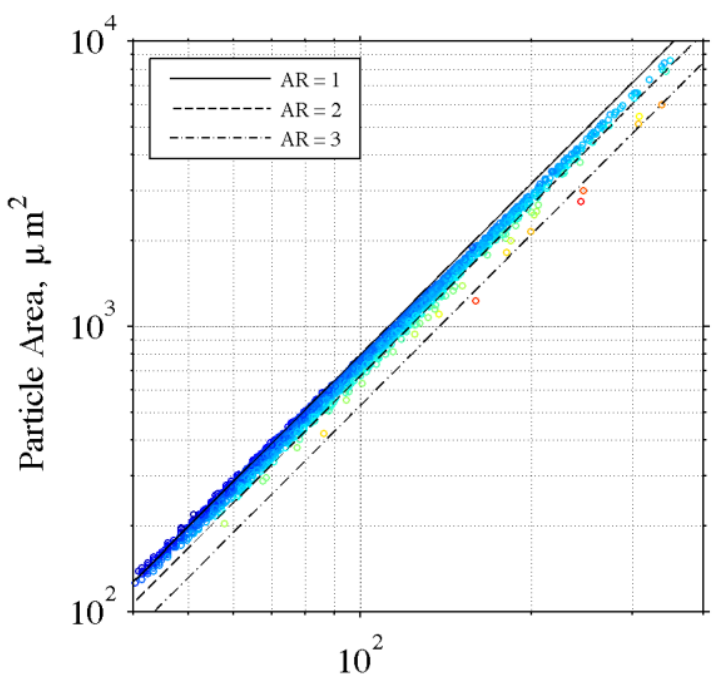

Particle Perimeter, $\mu \mathrm{m}$

(d)
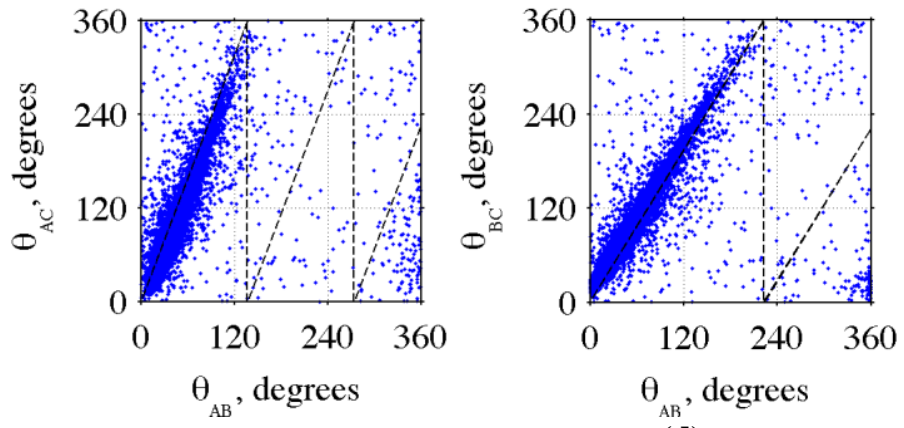

(d)

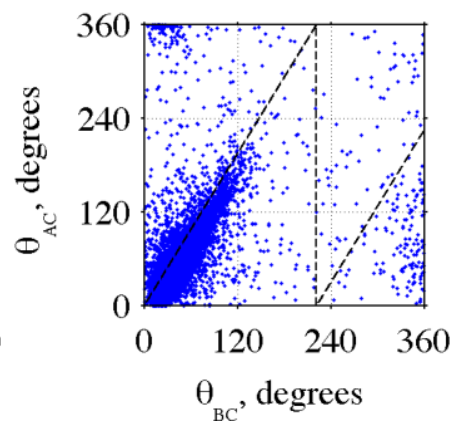

Figure 8. Test Point 201.1 Results showing (a) Cumulative Percent Volume Distribution PDI-HSI Comparison, (b) Number Density Distribution PDI-HSI Comparison, (c) Water Content Distribution PDI-HSI Comparison, (d) HSI Particle Area-Perimeter Correlation, and (d) PDI Raw Signal Phase Difference 


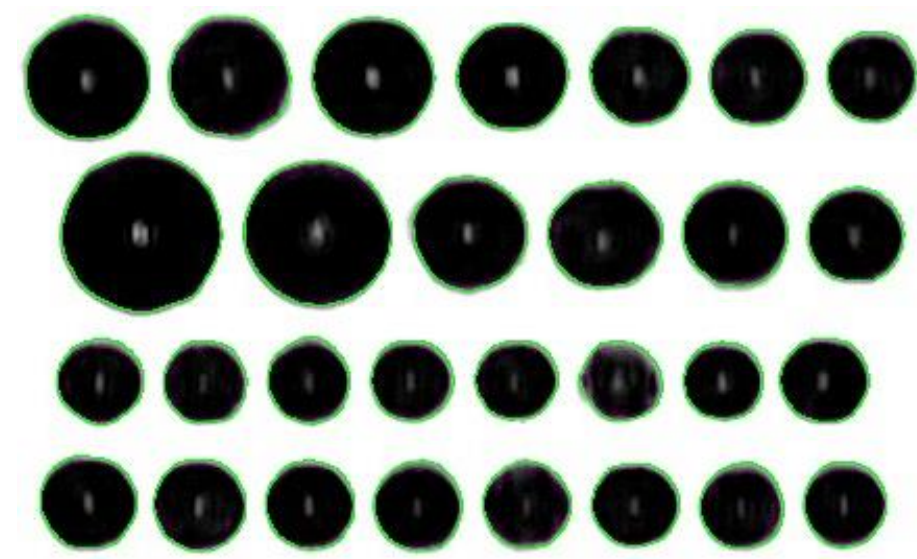

Figure 9. Test Point 210 sample particle images from the HSI

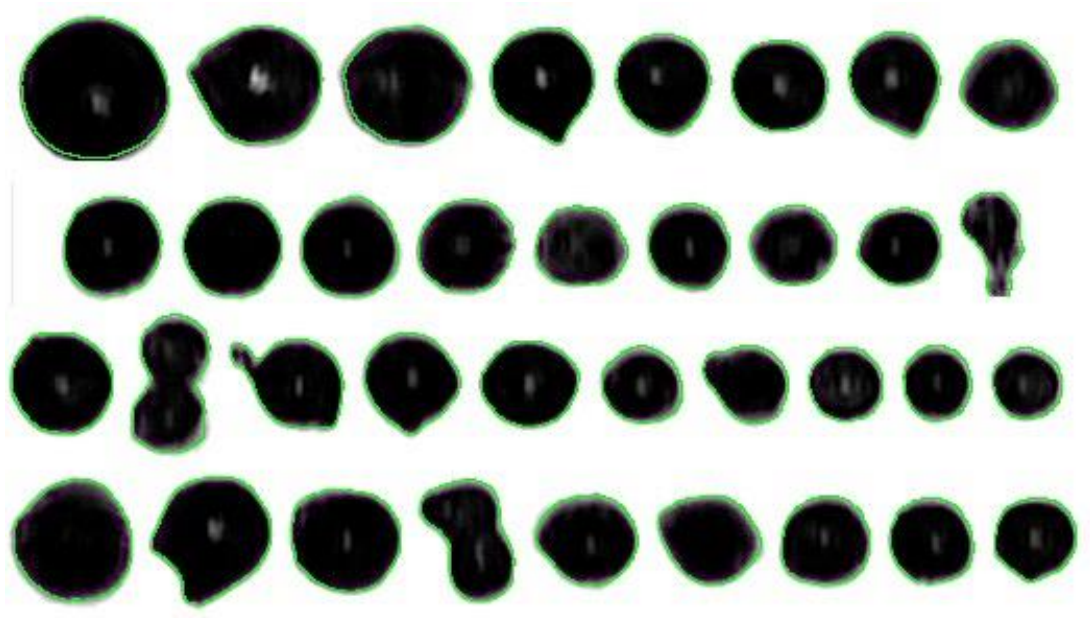

Figure 10. Test Point 201.1 sample particle images from the HSI 


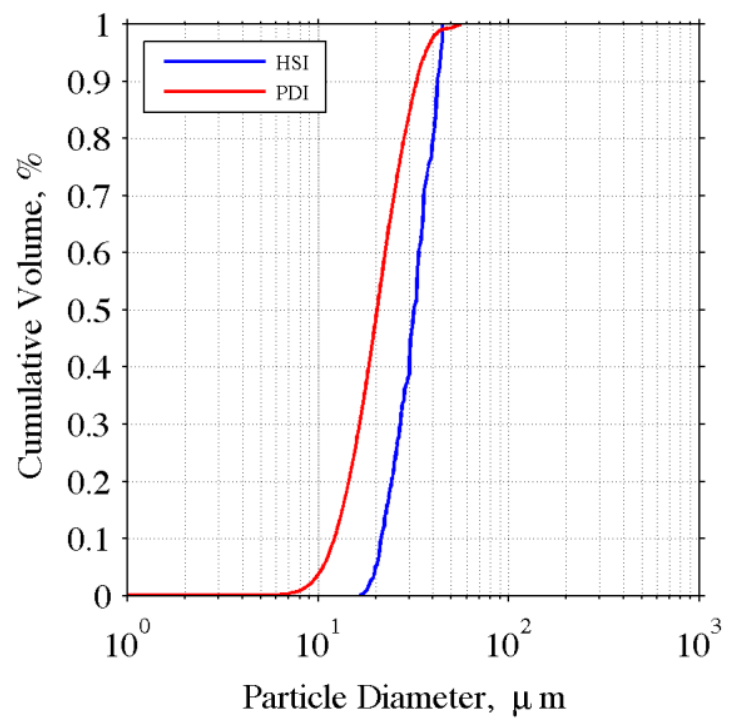

(a)

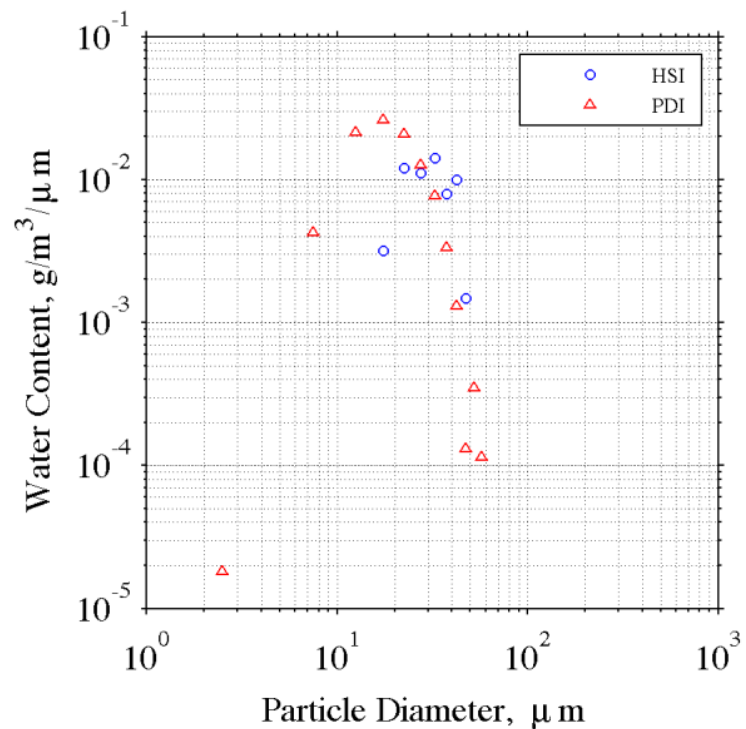

(c)

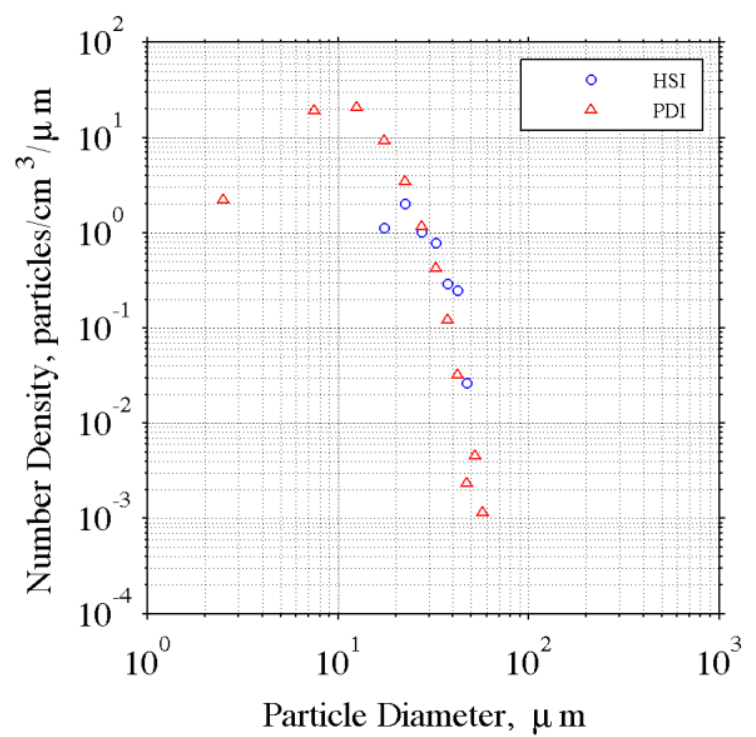

(b)

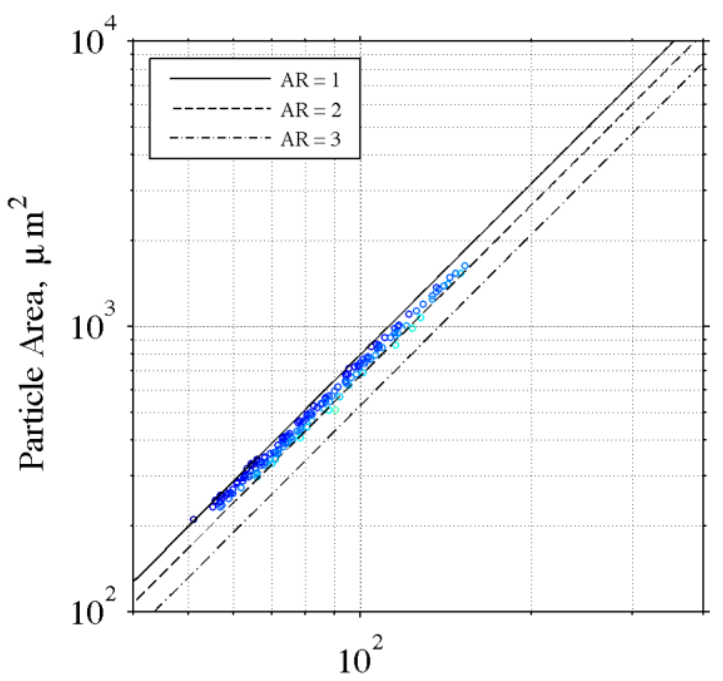

Particle Perimeter, $\mu \mathrm{m}$

(d)
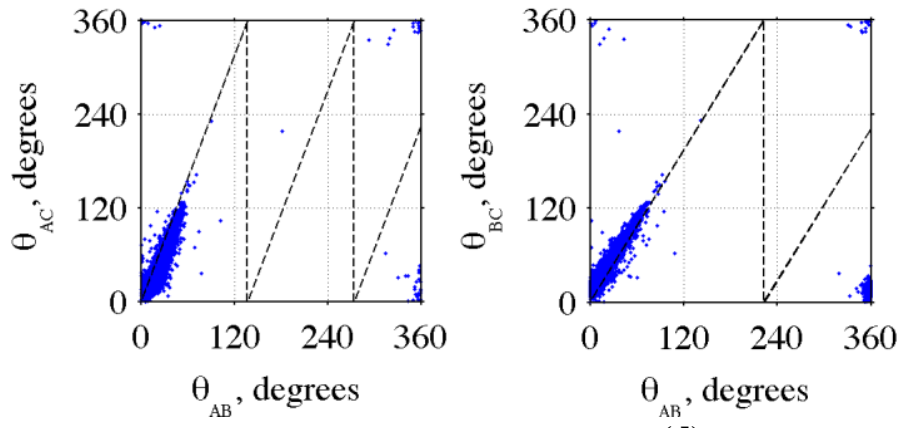

(d)

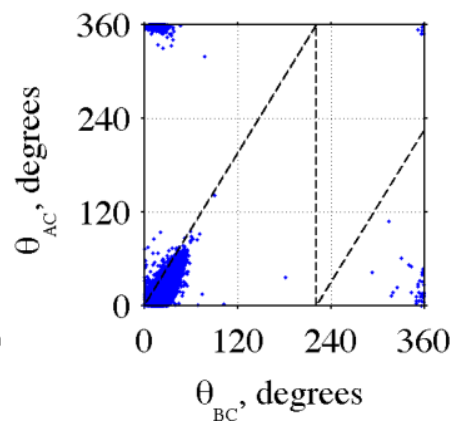

Figure 11. Test Point 211 Results showing (a) Cumulative Percent Volume Distribution PDI-HSI Comparison, (b) Number Density Distribution PDI-HSI Comparison, (c) Water Content Distribution PDI-HSI Comparison, (d) HSI Particle Area-Perimeter Correlation, and (d) PDI Raw Signal Phase Difference 


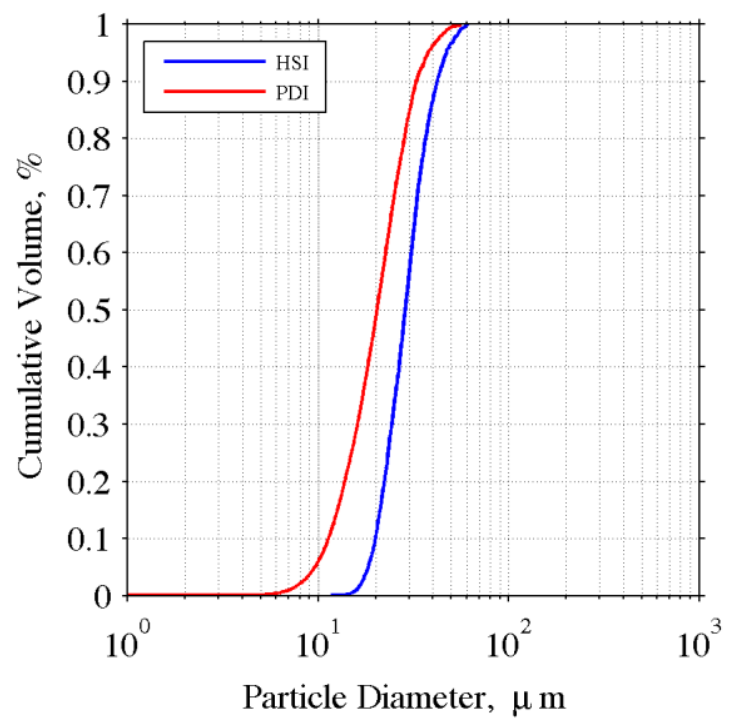

(a)

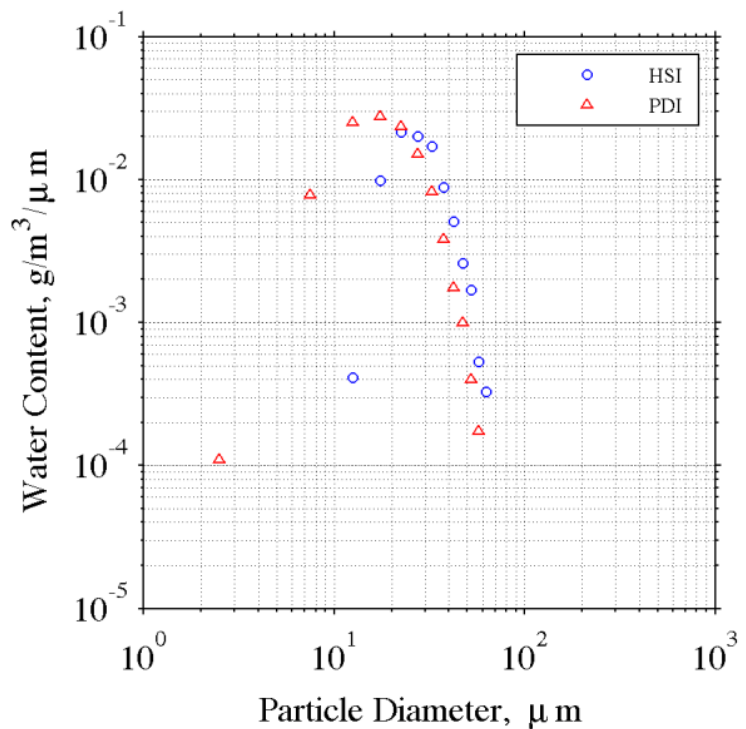

(c)

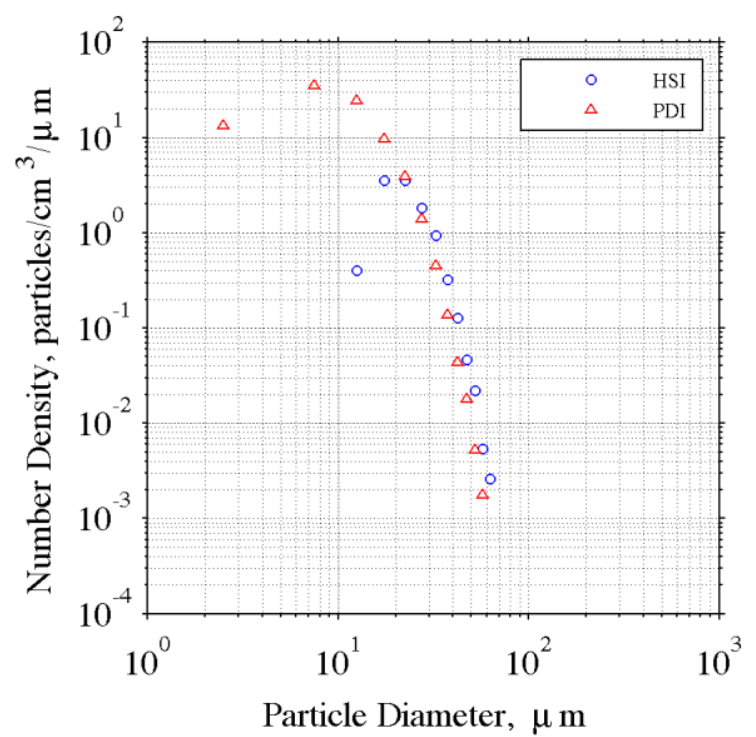

(b)

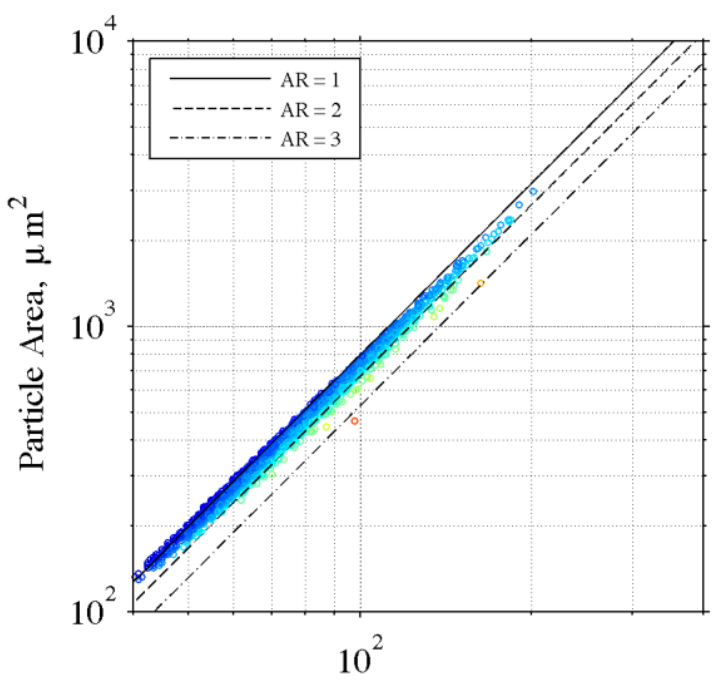

Particle Perimeter, $\mu \mathrm{m}$

(d)
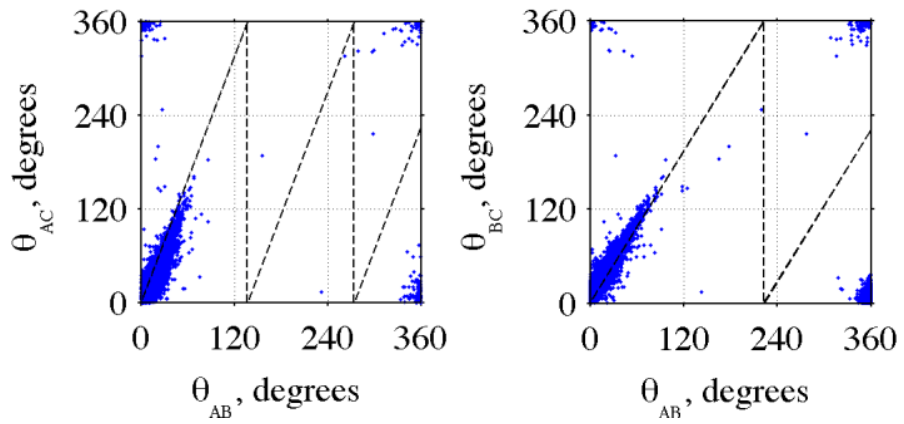

(d)

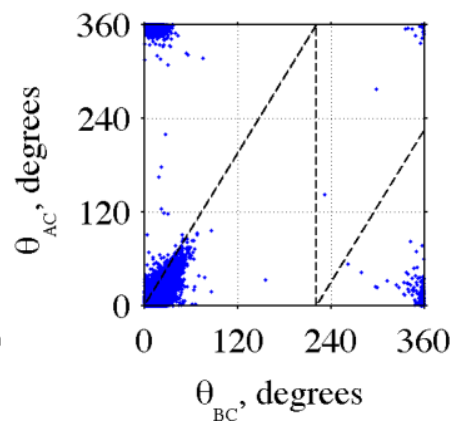

Figure 12. Test Point 208 Results showing (a) Cumulative Percent Volume Distribution PDI-HSI Comparison, (b) Number Density Distribution PDI-HSI Comparison, (c) Water Content Distribution PDI-HSI Comparison, (d) HSI Particle Area-Perimeter Correlation, and (d) PDI Raw Signal Phase Difference 


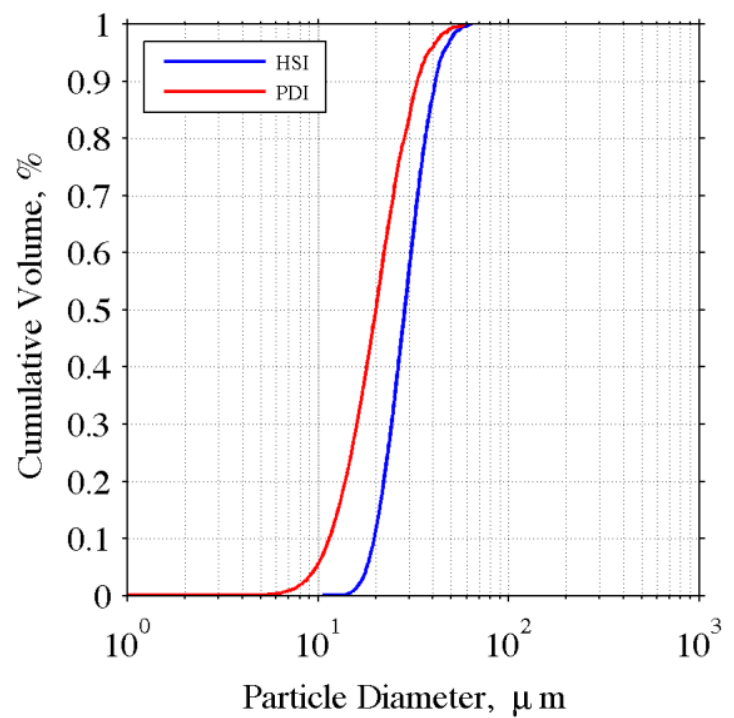

(a)

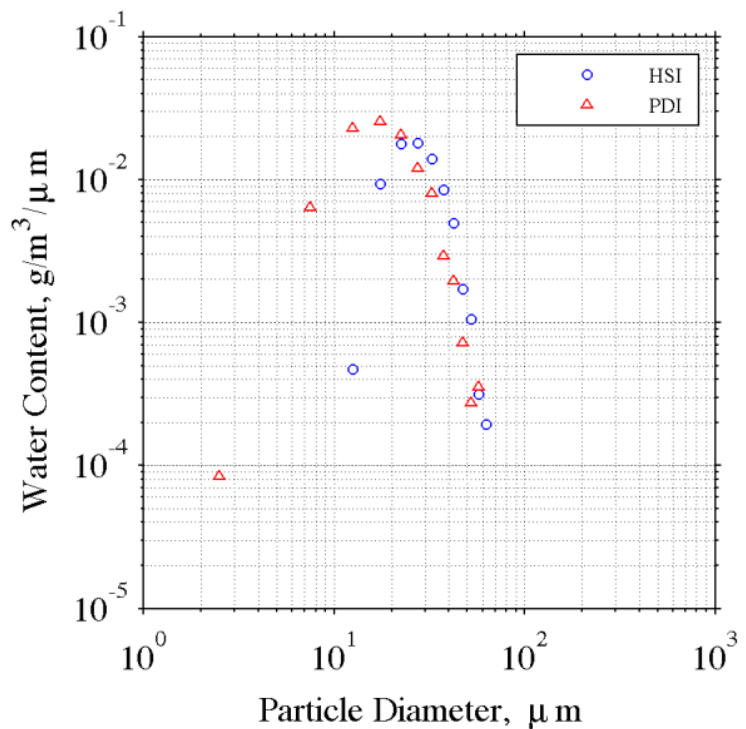

(c)

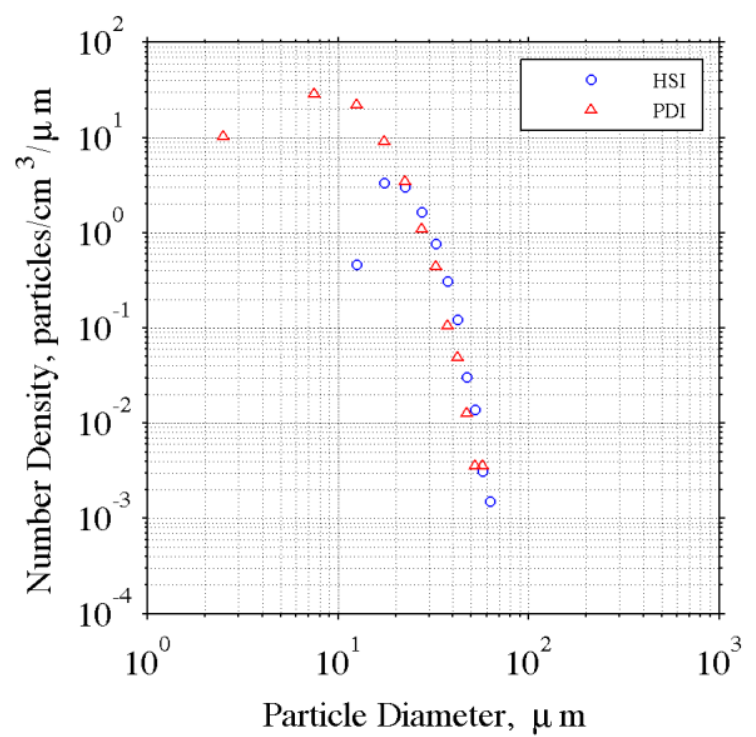

(b)

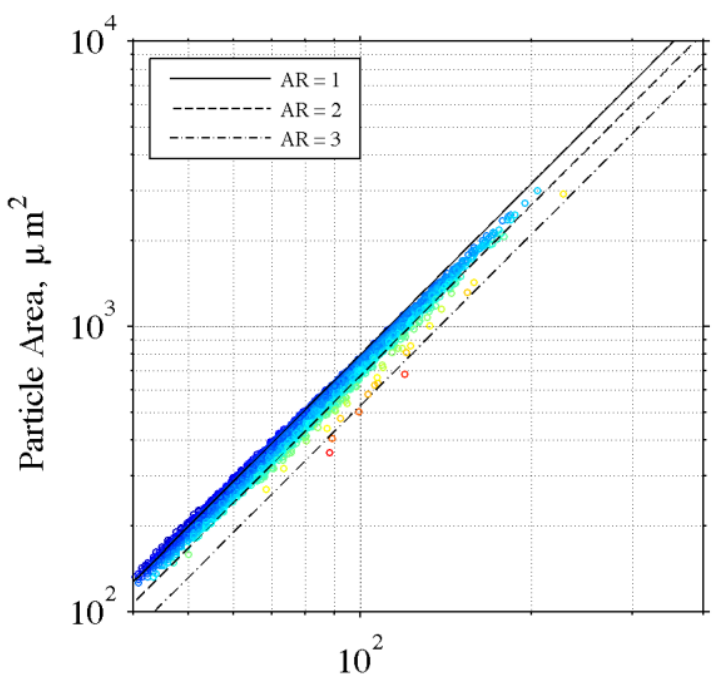

Particle Perimeter, $\mu \mathrm{m}$

(d)
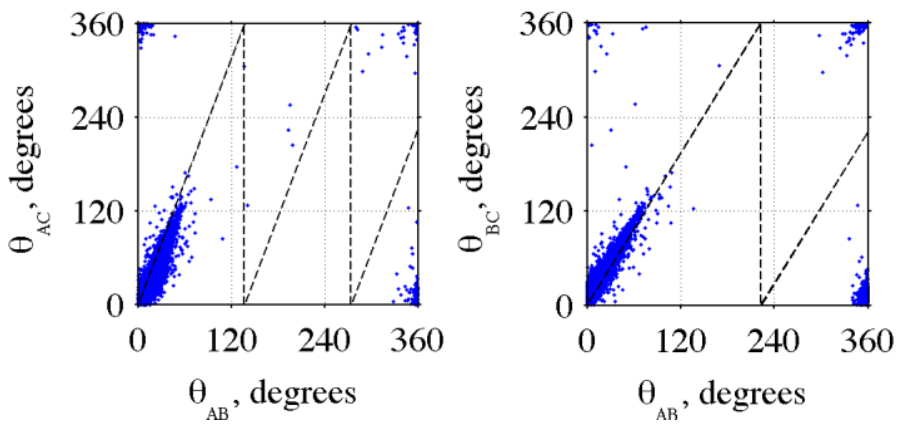

(d)

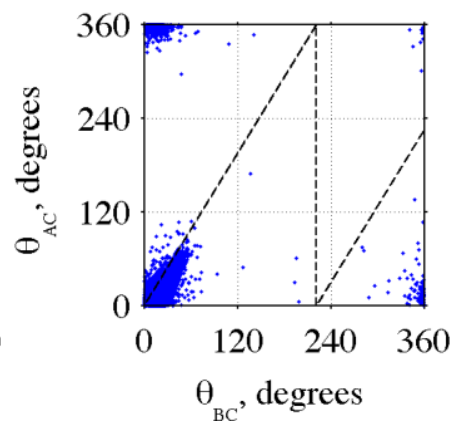

Figure 13. Test Point 207 Results showing (a) Cumulative Percent Volume Distribution PDI-HSI Comparison, (b) Number Density Distribution PDI-HSI Comparison, (c) Water Content Distribution PDI-HSI Comparison, (d) HSI Particle Area-Perimeter Correlation, and (d) PDI Raw Signal Phase Difference 


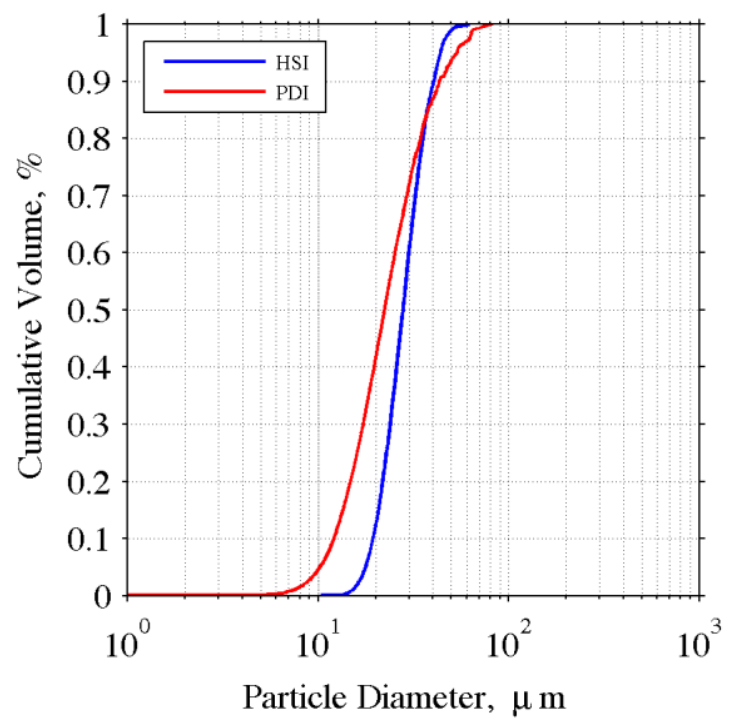

(a)

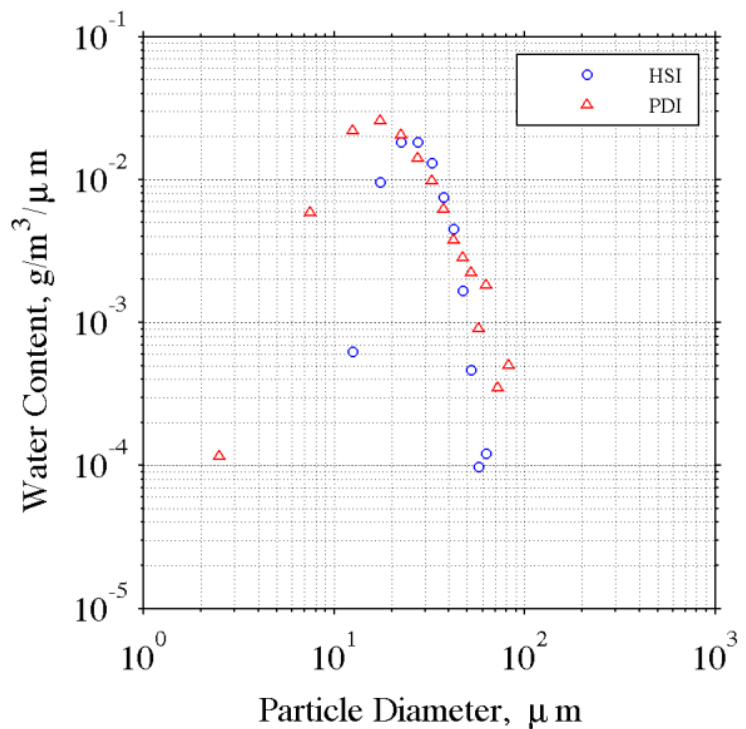

(c)

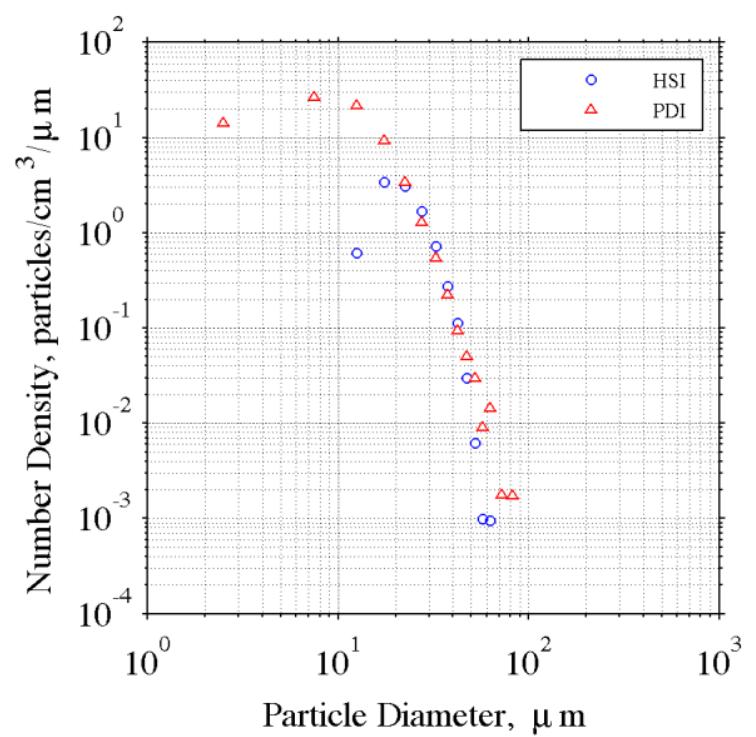

(b)

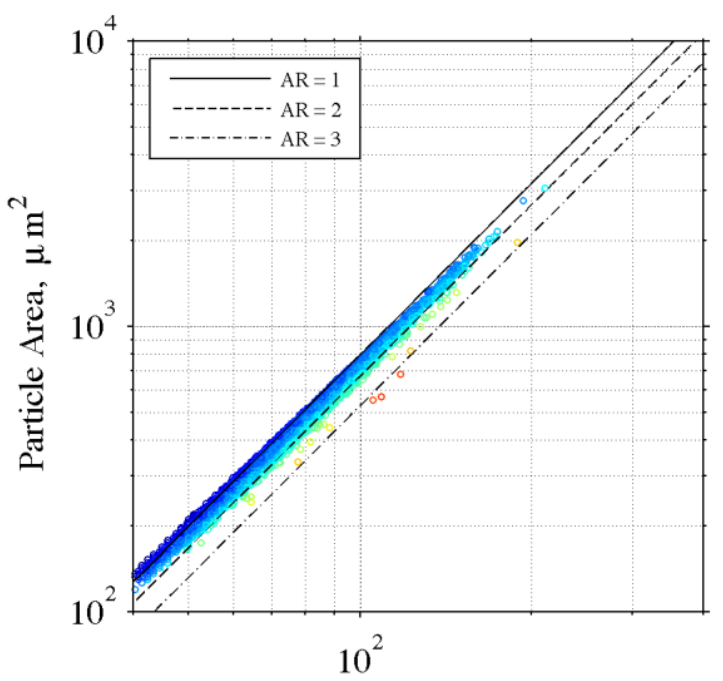

Particle Perimeter, $\mu \mathrm{m}$

(d)
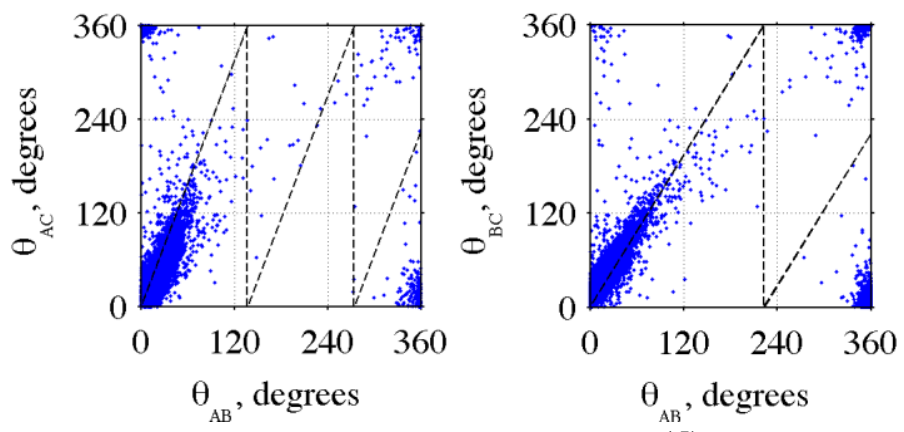

(d)

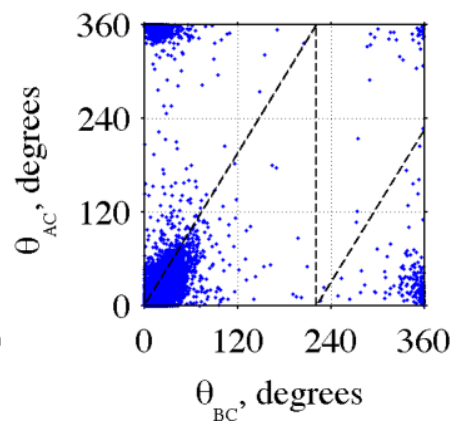

Figure 14. Test Point 207.1 Results showing (a) Cumulative Percent Volume Distribution PDI-HSI Comparison, (b) Number Density Distribution PDI-HSI Comparison, (c) Water Content Distribution PDI-HSI Comparison, (d) HSI Particle Area-Perimeter Correlation, and (d) PDI Raw Signal Phase Difference 


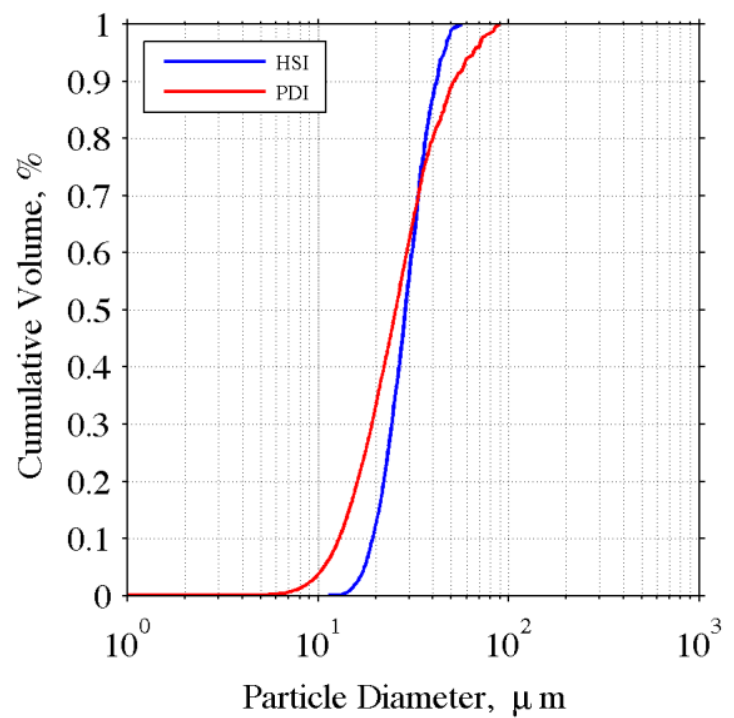

(a)

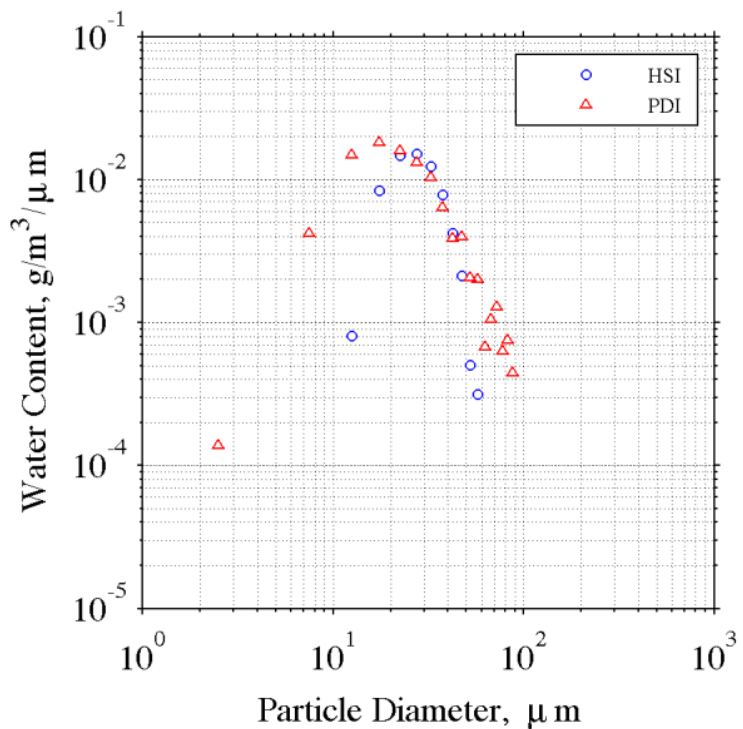

(c)

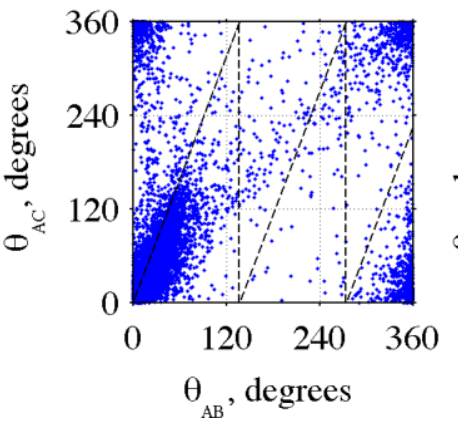

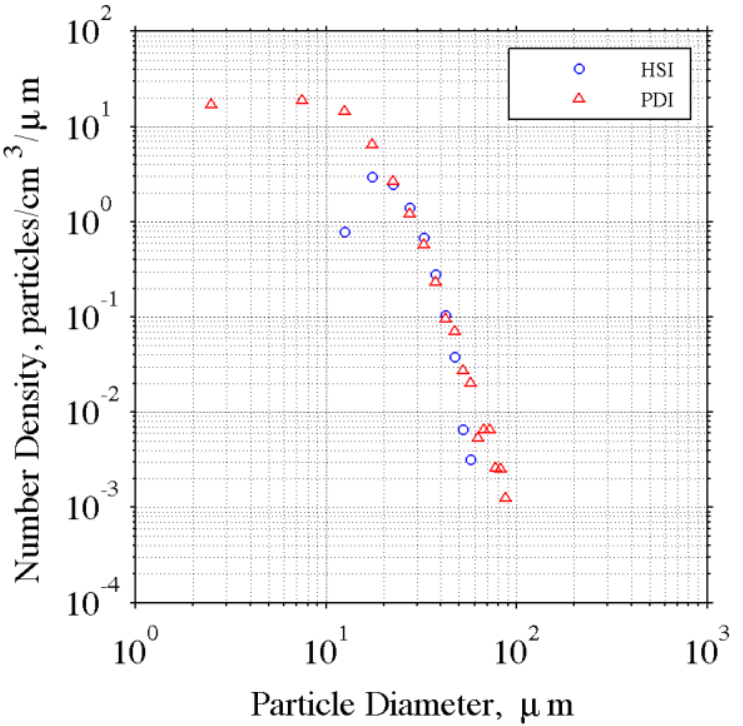

(b)

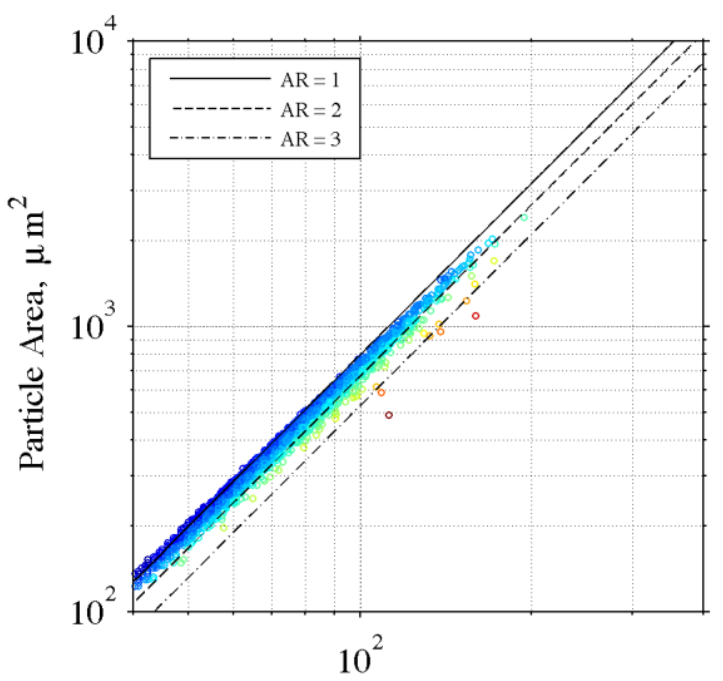

Particle Perimeter, $\mu \mathrm{m}$

(d)

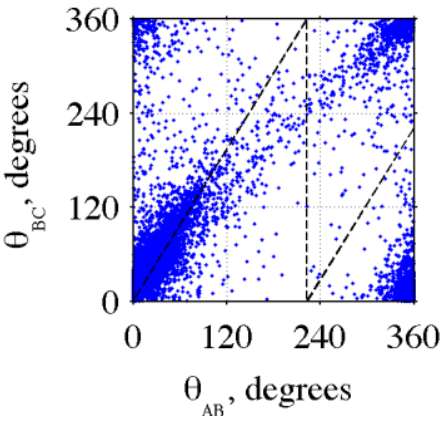

(d)

Figure 15. Test Point 205 Results showing (a) Cumulative Percent Volume Distribution PDI-HSI Comparison, (b) Number Density Distribution PDI-HSI Comparison, (c) Water Content Distribution PDI-HSI Comparison, (d) HSI Particle Area-Perimeter Correlation, and (d) PDI Raw Signal Phase Difference 


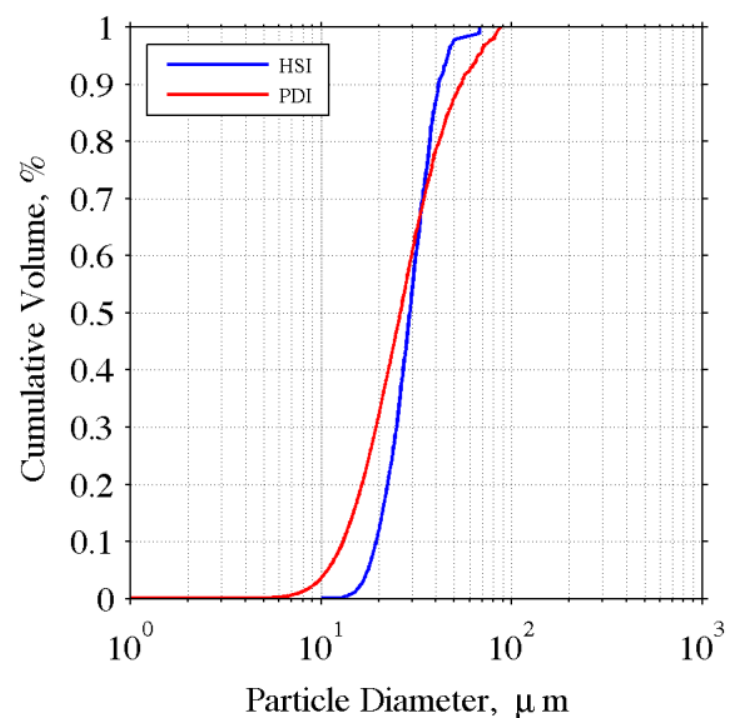

(a)

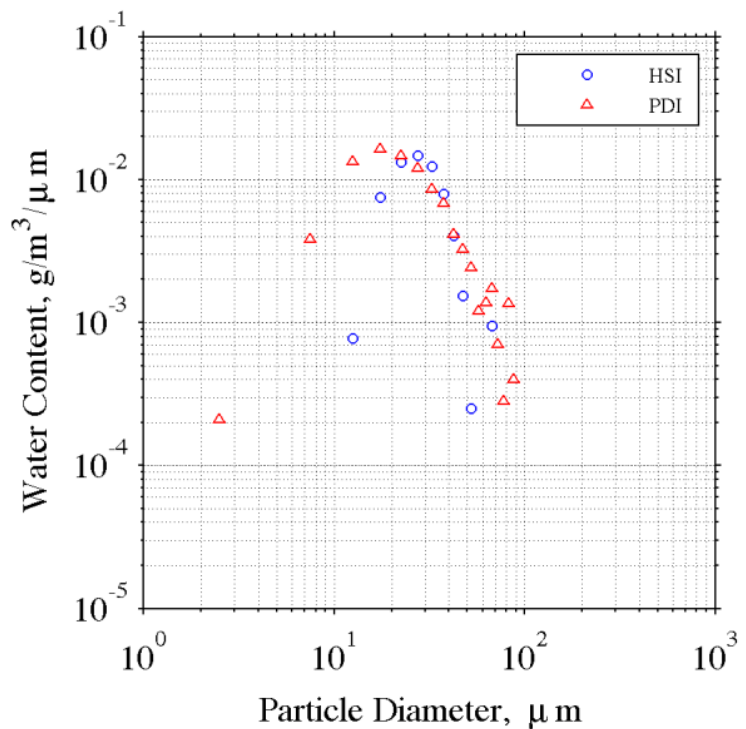

(c)

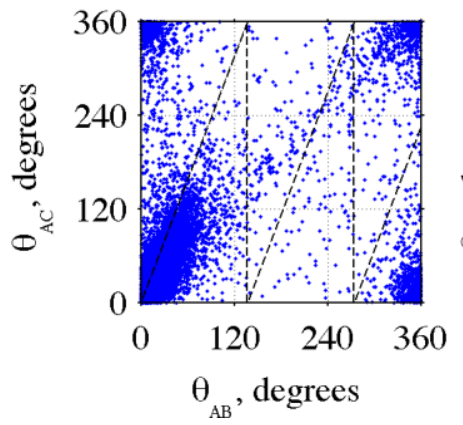

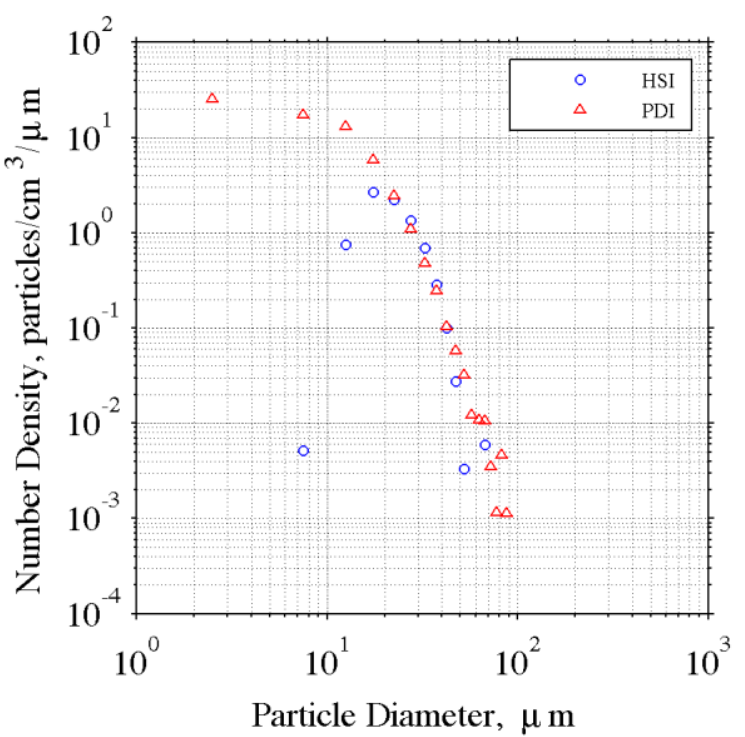

(b)

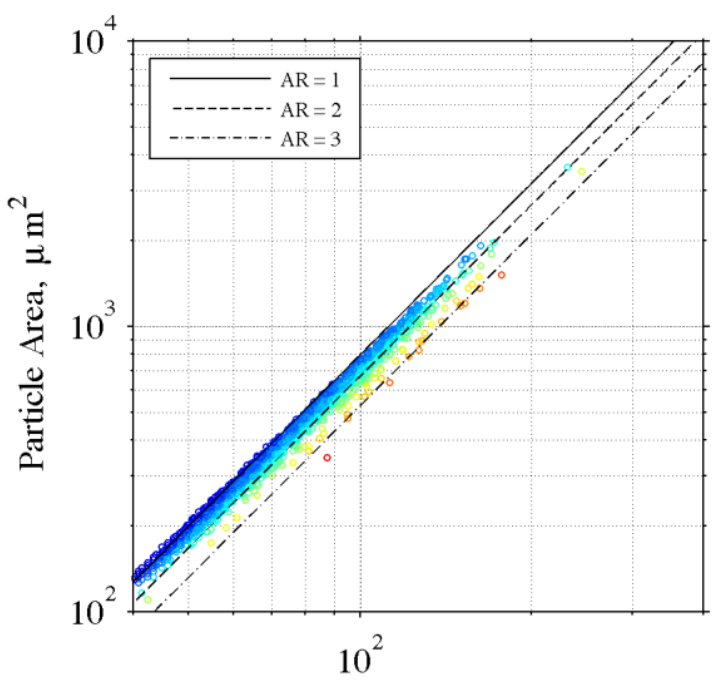

Particle Perimeter, $\mu \mathrm{m}$

(d)

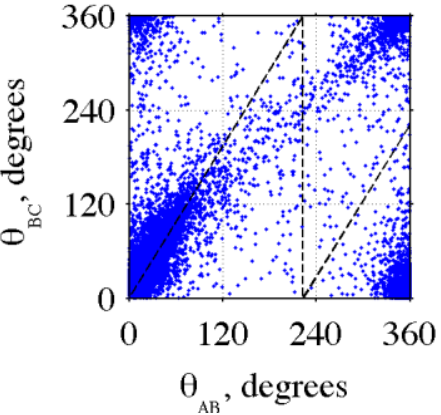

(d)

Figure 16. Test Point 203 Results showing (a) Cumulative Percent Volume Distribution PDI-HSI Comparison, (b) Number Density Distribution PDI-HSI Comparison, (c) Water Content Distribution PDI-HSI Comparison, (d) HSI Particle Area-Perimeter Correlation, and (d) PDI Raw Signal Phase Difference 


\section{0}

๑) 6อย

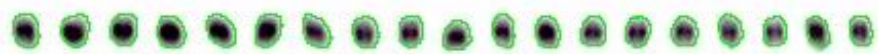

Figure 17. Test Point 211 sample particle images from the HSI

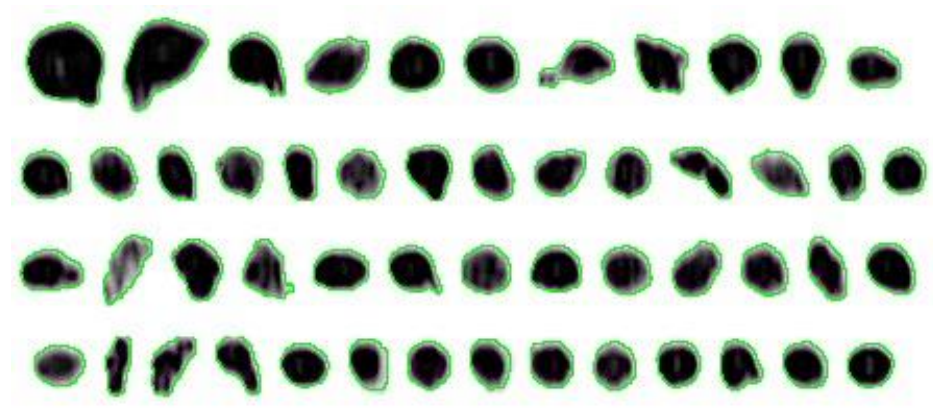

Figure 18. Test Point 203 sample particle images from the HSI 